\title{
Towards natural inflation in string theory
}

\author{
Ido Ben-Dayan, ${ }^{*}$ Francisco G. Pedro, ${ }^{\dagger}$ and Alexander Westphal ${ }^{\ddagger}$ \\ Deutsches Elektronen-Synchrotron DESY, Theory Group, D-22603 Hamburg, Germany
}

(Received 4 May 2015; published 13 July 2015)

\begin{abstract}
We provide type IIB string embeddings of two axion variants of natural inflation. We use a combination of RR 2-form axions as the inflaton field and have its potential generated by nonperturbative effects in the superpotential. Besides giving rise to inflation, the models developed take into account the stabilization of the compact space, an essential condition for any semirealistic model of string inflation.
\end{abstract}

DOI: $10.1103 /$ PhysRevD.92.023515

PACS numbers: 98.80.Cq, 04.65.+e

\section{INTRODUCTION}

Recent observational progress has drastically transformed cosmology into a quantitative science [1-4]. From these measurements we derive increasingly strong evidence for cosmological inflation, a very early epoch of accelerated expansion lasting for about $60 e$-foldings of the scale factor.

Recently, the BICEP2 Collaboration reported the first measurement of $B$-mode polarization of the cosmic microwave background (CMB) at large angular scales [5]. If this result stands after further corroboration and turns out to be primordial, then in the context of inflation it corresponds to a detection of primordial gravitational waves with a tensorto-scalar ratio $r=0.16_{-0.05}^{+0.06}$. Further results quantifying the polarized foreground emissions from e.g. galactic dust along the lines of [6-8] will help settle this question in the future.

The number of $e$-folds of slow-roll inflation

$$
N_{e}=\int_{\phi_{e}}^{\phi_{N_{e}}} \frac{d \phi}{\sqrt{2 \epsilon}}
$$

can be related to the tensor-to-scalar ratio, $r$, via the Lyth bound

$$
r=16 \epsilon \sim 0.003\left(\frac{50}{N_{e}}\right)^{2}\left(\frac{\Delta \phi_{N_{e}}}{M_{\mathrm{P}}}\right)^{2} .
$$

If we take the $B$-mode detection for the time being as signaling primordial inflationary tensor modes with $r \gtrsim 0.1$, then the Lyth bound $[9,10]$ tells us that the field excursion during inflation was super-Planckian: $\Delta \phi_{N_{e}} \gg M_{\mathrm{P}}{ }^{1}$

*ido.bendayan@desy.de

francisco.pedro@desy.de

*alexander.westphal@desy.de

${ }^{1}$ The field excursion can be reduced to sub-Planckian, if we allow for a nonmonotonic $\epsilon$ [11]. These types of models have received revived interest due to the BICEP2 result. We wish to note that there is no problem with having enough $e$-folds even with $\Delta \phi_{N_{e}} \ll 1$ and $n_{s} \simeq 0.96$ at the pivot scale per se. The actual limitation is that a smaller field excursion implies a faster change in $\epsilon$ which in turn implies a larger deviation from nearly scale invariance, which is harder to accommodate given the now $\simeq 7$ $e$-folds measured by PLANCK [11-13].
Inflation is known to be sensitive to the high-scale effects of a possible UV completion of the low energy theory; however, large and small field models are affected to different extents by this UV sensitivity. In small-field inflation $\Delta \phi_{N_{e}} \lesssim M_{\mathrm{P}}$ clearly requiring the tuning of dimension-6 operators to avoid $\mathcal{O}(1)$ contributions to the slow-roll parameter $\eta=V^{\prime \prime} / V$. To see this, note that a generic inflationary model will contain dimension- 6 operators of the type $\delta V_{6} \sim V_{0}(\phi) \phi^{2} / M_{\mathrm{P}}^{2}$. In a small-field model the startingpoint inflaton potential necessarily has the form

$$
\begin{aligned}
V_{0}(\phi) & =V_{0}\left(1+\sqrt{2 \epsilon_{0}} \frac{\phi}{M_{\mathrm{P}}}+\frac{\eta_{0}}{2} \frac{\phi^{2}}{M_{\mathrm{P}}^{2}}+\cdots\right) \simeq V_{0} \\
& =\text { const }
\end{aligned}
$$

at small field values $\phi \ll M_{\mathrm{P}}$. Hence, $\delta V_{6} \sim V_{0} \phi^{2} / M_{\mathrm{P}}^{2}$ corrects $\eta$ by an $\mathcal{O}(1)$ value, destroying slow roll.

Large-field inflation in contrast is UV sensitive to an infinite series of dangerously irrelevant operators. Clearly, we must appeal to a protective symmetry to save the inflation direction in the scalar potential. This by definition will almost amount to an effective shift symmetry which is broken at leading order by the inflationary scalar potential itself. While this notion of a protective shift symmetry in large-field inflation is certainly natural in the bottom-up Wilsonian sense (corrections from self-interactions of inflaton fluctuations die out at large field values, and quantum Einstein gravity correction scale $\sim V / M_{\mathrm{P}}^{4}, V^{\prime \prime} / M_{\mathrm{P}}^{2} \ll 1$ ), realizing such a shift symmetry and establishing control over its breaking clearly requires high-scale information mandating the embedding into a theory of quantum gravity.

At present there is not a unique and well understood theory of quantum gravity. However, string theory constitutes the most prominent candidate, with many nontrivial intricate results concerning its mathematical structure, the right low-energy field content to potentially accommodate our local universe, and a successful microscopic description of a large part of black hole physics. This provides a clear motivation to study inflation and in particular its large-field varieties in string theory. The requirement of realizing a well-respected shift symmetry typically leads us to consider 
the many string theory axions from higher-dimensional $p$-form gauge fields, or their mirror-dual partners of complex structure moduli, as good inflaton candidates. However, many sectors of the theory display a periodicity under shift of the $p$-form axionic fields $a_{(p)} \rightarrow a_{(p)}+2 \pi$ while the kinetic terms of these axions

$$
\mathcal{L}_{\text {kin }}=f^{2}\left(\partial_{\mu} a_{(p)}\right)^{2}
$$

imply

$$
f \sim \frac{M_{\mathrm{P}}}{L^{p}} \ll M_{\mathrm{P}}
$$

for $10 d$ to $4 d$ compactifications with volume $\mathcal{V}=L^{6}$ in string units. The periodicity range of the canonically normalized axion field

$$
\frac{\phi^{(p)}}{M_{\mathrm{P}}}=\frac{f}{M_{\mathrm{P}}} a_{(p)} \sim \frac{a_{(p)}}{L^{p}} \lesssim 1
$$

is sub-Planckian in controllable compactifications requiring large volume and weak string coupling [14]. This discrete shift symmetry with sub-Planckian period is broken by the presence of quantized $p$-form fluxes or (dual) brane configuration, which unwraps the discrete shift symmetry into a system of multiple nonperiodic branches: the full system with fluxes or branes shows monodromy in the potential energy of the axion on each branch, while periodicity is retained when summing over multiple branches [15-26].

An alternative to monodromy proper arises in the presence of at least two axions [27-36]. Nonperturbative effects provide cosine potentials with typical sub-Planckian periodicities $2 \pi f_{1}$ and $2 \pi f_{2}$,

$$
\begin{aligned}
V= & \Lambda_{1}^{4}\left[1-\cos \left(\frac{p_{1}}{f_{1}} \phi_{1}^{(p)}+\frac{p_{2}}{f_{2}} \phi_{2}^{(p)}\right)\right] \\
& +\Lambda_{2}^{4}\left[1-\cos \left(\frac{q_{1}}{f_{1}} \phi_{1}^{(p)}+\frac{q_{2}}{f_{2}} \phi_{2}^{(p)}\right)\right] .
\end{aligned}
$$

An alignment of the $p_{i}, q_{i}$ [27] or a hierarchy like e.g. $q_{1} \ll$ $p_{1}, q_{2}$ with $p_{2}=0[32,33]$ (a fully nonperturbative variant of [28]) drives the emergence of a mass hierarchy in the axionic sector, which is translated into the appearance of an effective super-Planckian axion decay constant $f_{\text {eff }} \gg$ $M_{\mathrm{P}} \gtrsim f_{1}, f_{2}$. The arising effective single-field inflaton potential realizes the original idea of natural inflation [37].

A crucial step for any construction of large-field inflation in string theory consists of showing the compatibility of the shift symmetry and field-range extension mechanism with the process of moduli stabilization. The moduli potential tends to backreact on the inflationary vacuum energy. This generates corrections to the inflaton potentials, which energetically lead generically to the flattening of a naive potential shape as inferred from the pure large-field mechanism itself [38]. Generically, the inflationary vacuum energy may very well participate in moduli stabilization which often can enhance the stability of the compactification while showing the flattening effect $[26,38]$. If we restrict ourselves to the supersymmetric setup provided by Calabi-Yau (CY) flux compactification, stabilization of the volume moduli often requires nonperturbative effects. For this reason, CY compactifications often lead to problems with having the inflationary vacuum energy participating in moduli stabilization, requiring a (moderate) separation of scales between the moduli potential and the inflaton sector. This, however, is an artifact of our restricting to CY compactifications in the first place.

In this paper we are discussing several methods of embedding an aligned or hierarchical axion potential of the type (1.7) into type IIB string theory compactified on CY manifolds with 3-form flux fixing the complex structure moduli and the axio-dilaton [39]. Because of the arguments discussed above, and those expressed in Sec. IV A, we will restrict ourselves to the use of 2-form R-R sector axions $C_{2}$ which provide a rather well-protected shift symmetry in the context of type IIB on CY orientifolds with $\mathrm{O} 7$ planes and D3/D7-branes [16,40,41].

The paper is organized as follows. In Sec. II, we discuss natural inflation models in the two axions case. We will demonstrate that $[27,28,32,33]$ actually stem from the same origin. We embed such models in a supergravity framework in Sec. III. In Sec. IV we discuss their string theoretic derivation. We will study both the Kachru-Kallosh-LindeTrivedi (KKLT) mechanism [42] and the large-volume scenario [43] for combining Kähler moduli stabilization with nonperturbative effects from gaugino condensation on D5-branes or ED3-ED1 instanton from Euclidean D3branes providing the axion potential for the $2 \mathrm{R}-\mathrm{R}$ sector $C_{2}$ axions. Finally we conclude in Sec. V.

\section{NATURAL INFLATION FROM TWO AXIONS}

\section{A. A common origin}

In this section we discuss the two mechanisms that generate effective super-Planckian decay constants from fundamental sub-Planckian ones, following the works $[27,32,33]$. We show that actually both models (as well as [28]) come from the same origin and only correspond to different deformations of the underlying potential, or different breaking of the same shift symmetry.

Consider a two axion potential of the form

$$
\begin{aligned}
V= & \Lambda_{1}^{4}\left(1-\cos \left(\frac{p_{1}}{\tilde{f}_{1}} \phi_{1}+\frac{p_{2}}{\tilde{f}_{2}} \phi_{2}\right)\right) \\
& +\Lambda_{2}^{4}\left(1-\cos \left(\frac{q_{1}}{\tilde{f}_{1}} \phi_{1}+\frac{q_{2}}{\tilde{f}_{2}} \phi_{2}\right)\right),
\end{aligned}
$$

where the axions $\phi_{1}$ and $\phi_{2}$ have canonical kinetic terms and all decay constants are sub-Planckian: $\frac{\tilde{f}_{1}}{p_{1}}, \frac{\tilde{f}_{2}}{p_{2}}, \frac{\tilde{f}_{1}}{q_{1}}, \frac{\tilde{f}_{2}}{q_{2}}<M_{P}$. 
We start our discussion by imposing an alignment condition on the above scalar potential which ensures the presence of flat direction by arranging for $V=V\left(\phi_{1}+\phi_{2}\right)$. Then the determinant of the second derivative matrix vanishes everywhere, signaling the possibility of a flat direction det $V_{i j}=0 .{ }^{2}$ Hence all that is left is to find a region where the gradient $V_{i}$ is small, and we have the flat region desired for inflation, provided that the other eigenvalues of $V_{i j}$ are positive. This happens naturally at the origin of field space in (2.1). Slow-roll inflation requires deforming the flat direction slightly. We can achieve this in two nonequivalent directions away from the flat limit - either we relax the alignment condition in the spirit of Kim-Nilles-Peloso (KNP) or we introduce a subdominant scalar potential providing the slowly varying deformation away from flatness as in Dante's inferno and hierarchical axion inflation. We will now discuss both possibilities in more detail.

\section{B. KNP}

We start by discussing the KNP mechanism. As was mentioned above, in the perfect alignment limit $\frac{p_{2}}{p_{1}}=\frac{q_{2}}{q_{1}}$ the mass matrix is singular. With inflation model building in mind one deforms this condition to allow for small misalignment,

$$
\frac{p_{2}}{p_{1}} \equiv r \quad \text { and } \quad \frac{q_{2}}{q_{1}} \equiv r(1+\delta) \quad \text { with } \quad \delta \ll 1 .
$$

This has the effect of lifting the flat direction in a way suitable for slow-roll inflation provided $\delta$ is sufficiently small. One must note that for sufficiently small $\delta$, the alignment mechanism holds for generic values of the various parameters in Eq. (2.1), as can be seen from the fact that the determinant of the mass matrix

$$
\operatorname{det} M^{2} \equiv m_{1}^{2} m_{2}^{2}=\Lambda_{1}^{4} \Lambda_{2}^{4} \frac{p_{1}^{2} q_{1}^{2} r^{2}}{\tilde{f}_{1}^{2} \tilde{f}_{2}^{2}} \delta^{2}
$$

becomes singular in the limit of perfect alignment $(\delta=0)$, signaling the presence of a flat direction.

To leading order in $\delta$, the mass eigenvalues are

$$
\begin{aligned}
& m_{1}^{2}=\frac{p_{1}^{2} q_{1}^{2} r^{2}}{\tilde{f}_{2}^{2}+\tilde{f}_{1}^{2} r^{2}} \frac{\Lambda_{1}^{4} \Lambda_{2}^{4}}{p_{1}^{2} \Lambda_{1}^{4}+q_{1}^{2} \Lambda_{2}^{4}} \delta^{2} \quad \text { and } \\
& m_{2}^{2}=\frac{\tilde{f}_{2}^{2}+\tilde{f}_{1}^{2} r^{2}}{\tilde{f}_{1}^{2} \tilde{f}_{2}^{2}}\left(p_{1}^{2} \Lambda_{1}^{4}+q_{1}^{2} \Lambda_{2}^{4}\right),
\end{aligned}
$$

\footnotetext{
${ }^{2}$ In mathematics det $V_{i j}=0$ is called the Monge-Ampère equation. Starting from such an equation is much more general than our discussion here. The Monge-Ampère equation provides an excellent starting point for model building and model classification. We intend to return to this in future work.
}

which keeping in mind that the axions' masses are of the form $\Lambda^{4} / f^{2}$ implies that the large effective decay constant, corresponding to the eigenvalue $m_{1}^{2}$, scales as

$$
f_{\mathrm{eff}}^{2}=\frac{\tilde{f}_{2}^{2}+\tilde{f}_{1}^{2} r^{2}}{q_{1}^{2} r^{2} \delta^{2}}
$$

And so, by considering two almost aligned axions with originally sub-Planckian decay constants, one generates an effective super-Planckian decay constant as is required for natural inflation for the price of tuning an alignment of the original axion decay constants. This extension of the field range without requiring a super-Planckian fundamental domain constitutes the main advantage of this model, when compared with the original single-cosine realization of natural inflation [37].

\section{Hierarchical axions}

The hierarchical axions (HA) model (and its close cousin Dante's inferno) corresponds to setting $p_{2}=0$ in (2.1). By definition there is no alignment whatsoever, and the deformation of the potential that will allow for inflation corresponds to introducing a hierarchy in the decay constants by having $p_{1} \ll q_{1}$. The mass matrix at the global minimum is such that

$$
\operatorname{det} M^{2}=\Lambda_{1}^{4} \Lambda_{2}^{4} \frac{p_{1}^{2} q_{2}^{2}}{\tilde{f}_{1}^{2} \tilde{f}_{2}^{2}}
$$

so in the limit $p_{1} \rightarrow 0$ an exact shift symmetry is recovered and for $q_{1} \gg p_{1}$ the symmetry is broken such that inflation ends at a stable minimum at the origin, provided that

$$
\frac{\Lambda_{2}}{\Lambda_{1}}>\sqrt{\frac{p_{1}}{q_{1}}} .
$$

The effective axion masses in the limit $q_{1} \gg p_{1}$ are

$$
m_{1}^{2}=\frac{\Lambda_{1}^{4}}{\tilde{f}_{2}^{2}}\left(\frac{p_{1} q_{2}}{q_{1}}\right)^{2}, \quad m_{2}^{2}=\frac{\Lambda_{1}^{4} p_{1}^{2}}{\tilde{f}_{1}^{2}}+\Lambda_{2}^{4}\left(\frac{q_{1}^{2}}{\tilde{f}_{1}^{2}}+\frac{q_{2}^{2}}{\tilde{f}_{2}^{2}}\right),
$$

and so we see that the mass spectrum is hierarchical, with $m_{1} \ll m_{2}$. Integrating out the heavy mode results in a single effective axion potential with an effective decay constant,

$$
f_{\text {eff }}=\tilde{f}_{2} \frac{q_{1}}{q_{2} p_{1}} .
$$

The model generates super-Planckian effective decay constants similar to KNP, but replaces the tuned alignment with a hierarchy between the decay constants. It 
further keeps the entire inflationary analysis in a subPlanckian domain avoiding the functional fine-tuning necessary in large field models. The advantages of the model as laid out in [32] are threefold: utilizing (i) only nonperturbative effects, (ii) the smallest number of axions, and (iii) the least amount of tuning of the input parameters. These advantages make the model specifically tractable from the string theoretic point of view.

Both the KNP alignment and the hierarchical axions mechanism produce an effectively single-field inflaton potential of the form $V(\phi)=\Lambda_{\text {eff }}^{4}\left[1-\cos \left(\phi / f_{\text {eff }}\right)\right]$ with an effective enhanced field range $f_{\text {eff }} \gtrsim M_{\mathrm{P}}$. Hence, its observational predictions for the spectral index $n_{s}$ of curvature perturbation and the tensor-to-scalar ratio $r$ agree with those of natural inflation itself [31]. In particular, for $f_{\text {eff }} \gtrsim 10 M_{\mathrm{P}}$ the scalar potential in the $60 e$-fold range approaches that of $m^{2} \phi^{2}$ inflation with $n_{s}=1-2 / N_{e} \simeq$ 0.967 and $r=8 / N_{e} \simeq 0.13$, while for $f_{\text {eff }} \lesssim 5 M_{\mathrm{P}}$ the model becomes of small-field type with $n_{s}$ growing more red and leaving the Planck 95\% region, while the tensor-to-scalar ratio drops to (currently) unobservable levels.

\section{SUPERGRAVITY EMBEDDINGS}

In this section we provide explicit ways to build the field theory models $[27,32,33]$ described above into supergravity. The goal of this approach is to provide a stepping stone to the realization of these inflationary models in string compactifications of type IIB string theory which we present in Sec. IV.
The minimal model requires two chiral multiplets $X_{m} \equiv$ $b_{m}+i c_{m}$ whose dynamics are determined by the canonical Kähler potential

$$
K=\frac{1}{4}\left(X_{1}+\bar{X}_{1}\right)^{2}+\frac{1}{4}\left(X_{2}+\bar{X}_{2}\right)^{2}
$$

and the nonperturbatively generated superpotential

$$
W=W_{0}+A e^{-p_{1} X_{1}-p_{2} X_{2}}+B e^{-q_{1} X_{1}-q_{2} X_{2}} .
$$

In the case $p_{2} \neq 0$ one has the KNP alignment mechanism giving rise to a super-Planckian axion, while if one chooses from the start $p_{2}=0$ inflation will proceed through hierarchies in the parameters $p_{1}, q_{1}$, and $q_{2}$. The structure of the scalar potential will be similar in both cases, and so we analyze them simultaneously whenever possible.

The scalar potential can be made to have a hierarchy between the terms stabilizing the real parts of $X_{1}$ and $X_{2}$ and those generating the inflationary potential, thereby decoupling the heavy fields from the inflationary dynamics.

The $F$ term potential

$$
\begin{aligned}
V & =e^{K}\left(D_{I} W D^{I} \bar{W}-3|W|^{2}\right), \quad \text { where } \\
D_{I} W & =\partial_{I} W+W \partial_{I} K
\end{aligned}
$$

can be written as

$$
V=V_{0}+V_{1}+V_{2},
$$

where

$$
\begin{gathered}
V_{0}=e^{b_{1}^{2}+b_{2}^{2}} W_{0}^{2}\left(-3+2 b_{1}^{2}+2 b_{2}^{2}\right), \\
V_{1}=2 e^{b_{1}^{2}+b_{2}^{2}} W_{0}\left\{A F_{2}\left[p_{1}, p_{2}\right] \cos \left[p_{1} c_{1}+p_{2} c_{2}\right]+B F_{2}\left[q_{1}, q_{2}\right] \cos \left[q_{1} c_{1}+q_{2} c_{2}\right]\right\}
\end{gathered}
$$

and

$$
\begin{aligned}
V_{2}= & A^{2} F_{3}\left[p_{1}, p_{2}\right]+B^{2} F_{3}\left[q_{1}, q_{2}\right]+2 A B e^{-\left(p_{1}+q_{1}\right) b_{1}-\left(p_{2}+q_{2}\right) b_{2}+b_{1}^{2}+b_{2}^{2}} \cos \left[\left(p_{1}-q_{1}\right) c_{1}+\left(p_{2}-q_{2}\right) c_{2}\right] \\
& \times\left(-3+2\left(p_{1}-b_{1}\right)\left(q_{1}-b_{1}\right)+2\left(p_{2}-b_{2}\right)\left(q_{2}-b_{2}\right)\right) .
\end{aligned}
$$

For the sake of short formulas we have defined the $b_{1}$ and $b_{2}$ dependent quantities

$$
\begin{aligned}
& F_{2}[m, n] \equiv e^{-m b_{1}-n b_{2}}\left(-3+2 b_{1}\left(-m+b_{1}\right)+2 b_{2}\left(-n+b_{2}\right)\right), \\
& F_{3}[m, n] \equiv e^{-2 m b_{1}+b_{1}^{2}-2 n b_{2}+b_{2}^{2}}\left(-3+2\left(m-b_{1}\right)^{2}+2\left(n-b_{2}\right)^{2}\right) .
\end{aligned}
$$

Both in the KNP and in the hierarchical regimes we focus on regions of parameter space where $\left|W_{0}\right| \gg$ $\left|A e^{-p_{1} X_{1}}\right|,\left|A e^{-p_{1} X_{1}-p_{2} X_{2}}\right|,\left|B e^{-q_{1} X_{2}-q_{2} X_{2}}\right|$ so that one can stabilize $b_{1}, b_{2}$ at high scale before analyzing the inflationary potential. This hierarchy in the superpotential descends into the scalar potential: $V_{0} \gg V_{1} \gg V_{2}$. In these simple supergravity models the need for this hierarchy derives from the desire to analytically minimize the potential in a controlled way, and it is not a fundamental requirement of these models since we expect the alignment/ hierarchical mechanisms to produce a super-Planckian direction even in the absence of this hierarchy in $W$. 
This situation will change once we consider stringy embeddings of this idea, as we will see in Sec. IV, since this tuning will be related to parametric decoupling of the moduli vacuum.

We note in passing, that more generally parametric decoupling is an overly conservative criterion which may be relaxed in concrete string embeddings. The moduli potential may backreact appreciably during the inflaton evolution, which due to energetic reasons generically leads to a flattening of the inflaton potential expected from the parametrically decoupled limit [38]. In particular, if perturbative high-scale mechanisms serve to fix all the moduli, inflation may even participate and help with moduli stabilization allowing for significant yet controllable flattening effects [26]. The nonperturbative mechanisms of volume stabilization we use here are more sensitive to backreaction effects. This limits the amount of controllable flattening achievable and motivates us to restrict ourselves to the limit of parametric decoupling for the sake of explicitness.

Note that the combined minimum of the three cosine terms above and the moduli potential usually is an anti-de Sitter (AdS) vacuum. As discussed in many of the dS vacuum constructions in string theory in recent years, we need to add an uplifting contribution from e.g. an anti D3brane [42], D-terms with field-dependent Fayet-Iliopoulos (FI) terms $[44,45]$ or dilaton dependent nonperturbative effects [46]

$$
\delta V_{\text {uplift }}=\frac{C}{\mathcal{V}^{p}}, \quad \text { with } \quad p=\mathcal{O}(1)>0,
$$

to the scalar potential to lift the AdS minimum to a near Minkowski state $\left\langle V_{F}+\delta V_{\text {uplift }}\right\rangle \simeq 0$. Provided we already arranged for sufficient hierarchy between the moduli masses and the axion mass scales arising from the three cosine terms in $V_{F}$ in the prior AdS vacuum, this will survive an uplifting term of the above type. Hence, we will from now on tacitly assume the presence of such an uplifting term in our setups, which justifies the form $V(\phi) \sim 1-\cos (\phi / f)$ for the three cosine terms arising from the moduli potential.

The structure of the $F$-term potential, Eq. (3.3), implies that a three cosine potential is inevitable in supergravity, modifying Eq. (2.1) to

$$
\begin{aligned}
V= & \Lambda_{1}^{4}\left(1-\cos \left(\frac{p_{1}}{\tilde{f}_{1}} \phi_{1}+\frac{p_{2}}{\tilde{f}_{2}} \phi_{2}\right)\right)+\Lambda_{2}^{4}\left(1-\cos \left(\frac{q_{1}}{\tilde{f}_{1}} \phi_{1}+\frac{q_{2}}{\tilde{f}_{2}} \phi_{2}\right)\right) \\
& +\Lambda_{3}^{4}\left(1-\cos \left(\frac{p_{1}}{\tilde{f}_{1}} \phi_{1}+\frac{p_{2}}{\tilde{f}_{2}} \phi_{2}-\frac{q_{1}}{\tilde{f}_{1}} \phi_{1}-\frac{q_{2}}{\tilde{f}_{2}} \phi_{2}\right)\right) .
\end{aligned}
$$

Even though this will alter the expressions for the mass eigenvalues, which will receive $\Lambda_{3}$ dependent contributions, the existence of a mass hierarchy remains and the large effective decay constants are still given by Eqs. (2.5) and (2.9) for the KNP and HA cases, respectively.

Noting that $V_{0}$ depends only on the combination $y \equiv$ $b_{1}^{2}+b_{2}^{2}$ we see that the tree level action stabilizes $y$ at

$$
\langle y\rangle=1 / 2,
$$

and so $b_{1}, b_{2}$ must lie in a circle of radius $1 / \sqrt{2}$ centered at the origin of the $\left(b_{1}, b_{2}\right)$ plane. This is illustrated in Fig. 1. At this level there is still one flat direction left in the $\left(b_{1}, b_{2}\right)$ plane as well as two in the $\left(c_{1}, c_{2}\right)$. Since $V_{0}$ is independent from $p_{2}$, this result applies to both the KNP and the hierarchical axion mechanism. The flat directions will be lifted by the next-to-leading order contribution to the potential, $V_{1}$, which we analyze separately in the two regimes.

\section{A. KNP alignment mechanism}

We start by studying the vacua of $V_{1}$ in KNP in the limit of perfect alignment, $\frac{p_{2}}{p_{1}}=\frac{q_{2}}{q_{1}} \equiv r$, where we know there will be one unfixed direction in the $c$ plane. We will then follow
[27] and allow for a slight misalignment which will lift the remaining flat direction in a way suitable for inflation. This procedure is more subtle here than in the field theory case since the leading contribution to the potential, $V_{0}$ leaves the angular direction in the $\left(b_{1}, b_{2}\right)$ plane unfixed. One therefore has to ensure that by allowing for a slight misalignment in the decay constants in order to realize inflation, one is not simultaneously destabilizing the angular direction in the $\left(b_{1}, b_{2}\right)$.

In the alignment limit we have

$$
\begin{aligned}
V_{1}= & -4 W_{0} e^{\frac{1}{2} \frac{z\left(p_{1}+b_{1}\right)}{p_{1}}}\left(A e^{\frac{z q_{1}}{p_{1}}}(1+z) \cos \left[p_{1}\left(c_{1}+r c_{2}\right)\right]\right. \\
& \left.+B e^{z}\left(1+z \frac{q_{1}}{p_{1}}\right) \cos \left[q_{1}\left(c_{1}+r c_{2}\right)\right]\right),
\end{aligned}
$$

where for algebraic simplicity we have set $p_{1}=q_{1}$ and defined $z \equiv p_{1} b_{1}+p_{2} b_{2}$. This potential will simultaneously stabilize $z$ and the axionic combination $c_{1}+r c_{2}$. The minimum is located at $\langle z\rangle=0$ and $\left\langle c_{1}+r c_{2}\right\rangle=0$. This implies that the vacuum in the $b$ plane lies at the intersection of the circle $b_{1}^{2}+b_{2}^{2}=1 / 2$ and the straight line passing through the origin $p_{1} b_{1}+p_{2} b_{2}=0$. By construction, in this limit, the same linear combination 

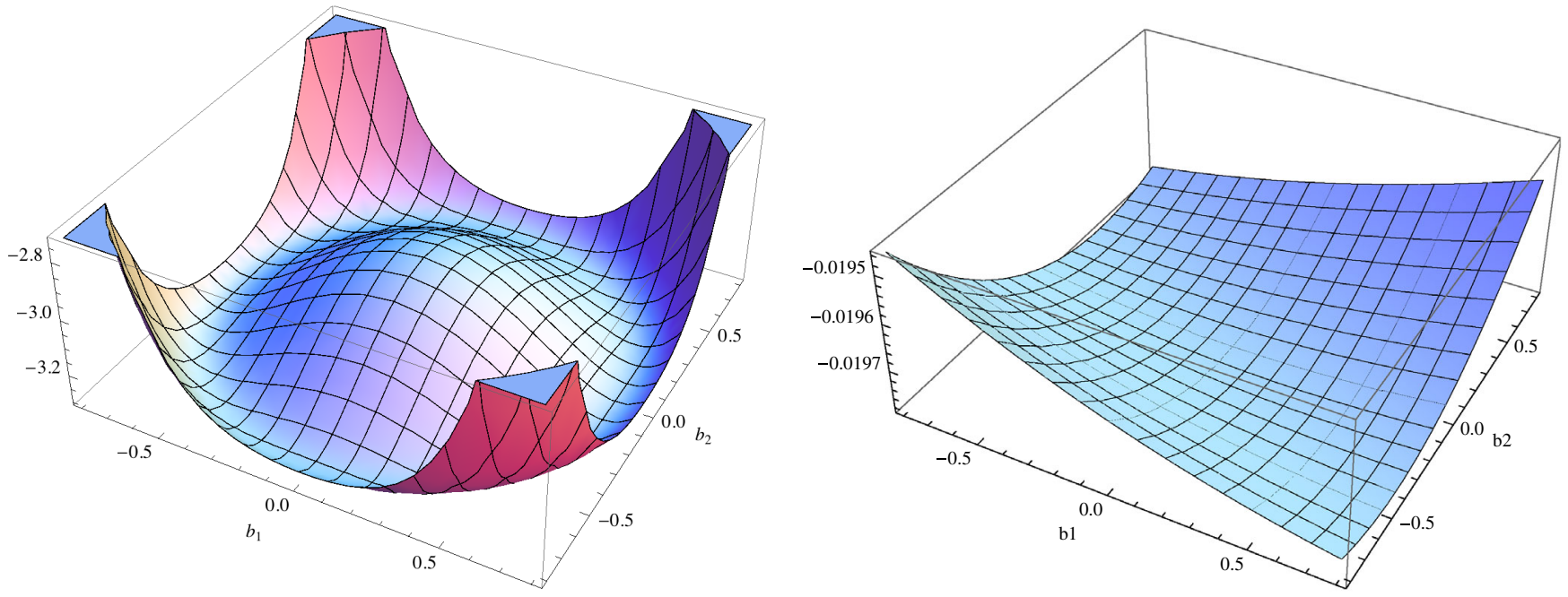

FIG. 1 (color online). Scalar potential in the $\left(b_{1}, b_{2}\right)$ plane for $\left\{A, B, W_{0}, r, \delta, b_{1}, a_{1}\right\}=\{0.002,0.001,1,2,0.1,0.1,0.05\}$. Right: $V_{0}$; left: $V_{1}$. Note the hierarchy between the two contributions to $V$.

of $c_{1}, c_{2}$ appears in the potential and so the orthogonal combination is exactly flat.

The introduction of a slight misalignment $\delta$ in the axionic decay constants,

$$
\frac{p_{2}}{p_{1}} \equiv r \quad \text { and } \quad \frac{q_{2}}{q_{1}} \equiv r(1+\delta),
$$

which will generate the inflationary potential, will also perturb the $b$ vacuum. This can in extreme cases lead to the destruction of the $b$ minimum or in more mild ones lead to a shift in the minimum's position. Since for inflationary purposes $\delta \ll 1$, the $b$ vacuum survives the introduction of a misalignment, with its position shifting by a small factor proportional to the smallness of the misalignment of the decay constants $\delta$,

$$
\langle z\rangle=-\delta \frac{B r p_{1} q_{1}^{2}}{\sqrt{2} \sqrt{1+r^{2}}\left(A p_{1}^{2}+B q_{1}^{2}\right)} .
$$

This fixes the remaining flat direction in the $b$ plane in the misaligned regime in a similar way to what was described before. The only difference is that the straight line intersecting the circle in the $b$ plane no longer passes through the origin. The effect of the misalignment in the stabilization of the real part of the fields $X_{1}$ and $X_{2}$ is therefore negligible.

The potential for the axions then admits the following expansion:

$$
\begin{aligned}
V_{1}= & -4 W_{0} \sqrt{e}\left\{A \cos \left[p_{1}\left(c_{1}+r c_{2}\right)\right]\right. \\
& \left.+B \cos \left[q_{1}\left(c_{1}+r(1+\delta) c_{2}\right)\right]\right\},
\end{aligned}
$$

which coincides with the field theory model of Eq. (2.1), with the identifications $\Lambda_{1}^{4}=4 W_{0} A \sqrt{e}$ and $\Lambda_{2}^{4}=4 W_{0} B \sqrt{e}$.

\section{B. Hierarchical axions mechanism}

Just as in the KNP case, for the hierarchical axion inflation model, the potential is generated by Eq. (3.6), but now with $p_{2}=0$. Anticipating that inflationary model building will require $q_{1} \gg q_{2}, p_{1}$ we can expand $V_{1}$ as

$$
\begin{aligned}
V_{1}= & -4 W_{0}\left(A \sqrt{e} \cos \left[p_{1} c_{1}\right]\right. \\
& \left.+B e^{1 / 2+z}(z-1) \cos \left[q_{1} c_{1}+q_{2} c_{2}\right]\right),
\end{aligned}
$$

where $z \equiv-q_{1} b_{1}-q_{2} b_{2}$.

$V_{1}$ is simultaneously responsible for stabilizing the angular direction in the $b$ plane (or equivalently stabilizing $z$ ) and giving rise to inflation. To leading order in a $q_{1}$ expansion, we find that $\langle z\rangle=0$, implying that the $b$ vacuum is the same as in the aligned KNP regime. In this case the potential then simplifies to the desired form

$$
V_{1}=-4 W_{0}\left(A \sqrt{e} \cos \left[p_{1} c_{1}\right]+B \sqrt{e} \cos \left[q_{1} c_{1}+q_{2} c_{2}\right]\right)
$$

One then concludes that the model of Eqs. (3.1) and (3.2) does indeed provide a supergravity description of the two axion versions of natural inflation of [27] and [32,33]. Generically this did not have to be the case since the structure of supergravity requires a model that initially involves four fields: two axions and two saxions/moduli, whose masses are closely linked. What we have shown is that the mechanism that allows for the generation of a potential suitable for inflation simultaneously guarantees that there is a mass hierarchy between the lightest axion and all the saxions/moduli. When trying to embed these models in string compactifications we will therefore be looking for the generic structures of (3.1) and (3.2), the adequate mass hierarchies, and tunable parameters. 


\section{NATURAL INFLATION IN STRING COMPACTIFICATIONS}

\section{A. Axions in string compactifications}

We confine our discussion to flux compactifications of type IIB string theory on $\mathrm{O} 3 / \mathrm{O} 7$ orientifolds of warped Calabi-Yau 3-folds with 3-form NS-NS and R-R flux. We assume a choice of 3-form fluxes such that they stabilize the complex structure moduli and the type IIB axio-dilaton supersymmetrically at a high mass scale while generating an effectively constant superpotential $W_{0}$ [39].

For the stabilization of the Kähler moduli we consider nonperturbative stabilization à la KKLT [42] involving gaugino condensation on D7-brane or D5-brane stacks or Euclidean D3-brane (ED3) instantons, or the large volume scenario (LVS) involving a combination of the leading $\mathcal{O}\left(\alpha^{13}\right)$ correction to the CY 3-volume and an ED3-brane instanton or D7-brane stack.

At the $\mathcal{N}=2$ level prior to imposing the $\mathrm{O} 7$ projection, the Kähler moduli sector consists of $h^{1,1}$ 2-cycle moduli $t^{j}=v^{j}+i b^{j}$. Here the $b^{j}=\int_{\Sigma_{2_{j}}} B_{2}$ denotes the NS-NS 2form axions $b^{j}$ arising from the NS 2-form $B$-field on the $h^{1,1} 2$-cycles $\Sigma_{2_{j}}$, while the $v_{j}$ denote the 2-cycle geometric volumes in string units. The tree-level Calabi-Yau volume $\mathcal{V}$ is then given by

$$
\mathcal{V}=\frac{1}{6} k_{i j k} v^{i} v^{j} v^{k}
$$

Imposing the $\mathrm{O} 7$ projections projects the Kähler moduli sector into an O7-even and odd subspaces with respective dimensions $h_{+}^{1,1}$ and $h_{-}^{1,1}=h^{1,1}-h_{+}^{1,1}$. Moreover, this forces a rearrangement of the real scalars into $a=$ $1 \ldots h_{+}^{1,1} 4$-cycle moduli

$$
T_{a}=\frac{1}{2} k_{a b c} v^{b} v^{c}+i \int_{\Sigma_{4}^{a}} C_{4}+\frac{1}{2(S+\bar{S})} k_{a \beta \gamma} G^{\beta}\left(G^{\gamma}+\bar{G}^{\gamma}\right)
$$

and $\alpha=1 \ldots h_{-}^{1,1} 2$-form axion multiplets

$$
G^{\alpha}=\bar{S} b^{\alpha}+i c^{\alpha},
$$

where the type IIB axio-dilaton is $S=e^{-\phi}+i C_{0}$ and we have O7-odd 2-form R-R and NS-NS axions

$$
c^{\alpha}=\int_{\Sigma_{2_{\alpha}}} C_{2} \quad \text { and } \quad b^{\alpha}=\int_{\Sigma_{2_{\alpha}}} B_{2} .
$$

For the case of a single Kähler modulus $h_{+}^{1,1}=1$ and $h_{-}^{1,1} \geq$ 1 odd 2-form axion moduli we can invert the relation between $T$ and $t$ and acquire $t\left(T, G^{\alpha}\right)$. This gives a $4 d$ $\mathcal{N}=1$ Kähler potential of the Kähler moduli (see e.g. [47])

$$
K=-3 \ln \left[T+\bar{T}+\frac{1}{2(S+\bar{S})} k_{1 \beta \gamma}(G+\bar{G})^{\beta}\left(G^{\gamma}+\bar{G}^{\gamma}\right)\right] .
$$

Guided by this example, we conjecture that for CY manifolds with a volume of Swiss-cheese form with $h_{+}^{1,1}>$ 1 Kähler moduli we may find cases where the Kähler potential takes the form (see e.g. [48])

$$
\begin{aligned}
K=-2 \ln \mathcal{V}, \text { with } \mathcal{V}= & c_{L}\left(T_{L}+\bar{T}_{L}+\frac{1}{2(S+\bar{S})} k_{L \beta \gamma}(G+\bar{G})^{\beta}\left(G^{\gamma}+\bar{G}^{\gamma}\right)\right)^{3 / 2} \\
& -\sum_{i=2 \ldots h_{+}^{1,1}} c_{i}\left(T_{i}+\bar{T}_{i}+\frac{1}{2(S+\bar{S})} k_{i \beta \gamma}(G+\bar{G})^{\beta}\left(G^{\gamma}+\bar{G}^{\gamma}\right)\right)^{3 / 2} .
\end{aligned}
$$

The Swiss-cheese type 4-cycle intersection numbers then are positive $c_{L}, c_{i}>0$. Because of the mixing between the $G^{\alpha}$ and $\tau$ in $K$ the results for the full $\left(T_{a}, G^{\alpha}, \tau\right)$-sector Kähler metric and its inverse are a bit lengthy. The full expressions are given in Eqs. (C.11) and (C.12) of Appendix C in [40].

We can now see from the form of e.g. Eq. (4.5) that the projection dictated by the $\mathrm{O} 7$ action leads to a breaking of the shift symmetry which the $B_{2}$ axions enjoyed at the four dimensional $\mathcal{N}=2$ level. If moduli stabilization proceeds via terms in the superpotential, then $W$ must be a holomorphic function of the chiral $\mathcal{N}=1$ superfields. Hence, superpotential stabilization of the moduli generically stabilizes the $T_{a}$ and $G^{\alpha}$ separately. The manifest dependence of $K$ on the $B_{2}$ axions being $\sim \operatorname{Im} G^{\alpha}$ hence breaks the shift symmetry of the $B_{2}$ axions. This fact renders the NS-NS 2form axions unsuitable for large-field inflation in these constructions.

If volume moduli stabilization had proceeded directly via stabilizing some of the geometric 4-cycle volumes e.g. by corrections to $K$, this could have restored the $B_{2}$ shift symmetry for the $G^{\alpha}$ involved. However, we will not pursue this opportunity here, and in our constructions the $G^{\alpha}$ will always appear in $W$ nonperturbatively.

This leaves the $C_{4}$ and $C_{2}$ R-R axions as potential inflaton candidates. Nonperturbative stabilization à la KKLT of the $T_{a}$ Kähler moduli then implies that the $C_{4}$ axions $\sim \operatorname{Im} T_{a}$ acquire the same mass scales as their 
moduli partners $\operatorname{Re} T_{a}$. In the LVS we require only a fraction of the $T_{a}$ to appear in $W$ via nonperturbative effects. For those which do not appear in $W$ but get fixed by Kähler corrections, this allows one to split the mass scale of their $C_{4}$-axion partners from their moduli mass scale [49]. In such cases, we may use $C_{4}$ axions as inflaton candidates.

Generically, however, we find the $C_{2}$ axions are least coupled to the process of volume stabilization regardless whether this proceeds nonperturbatively in $W$ or perturbatively in $K$. Hence, we focus on 2-axion models of largefield inflation driven by the R-R $C_{2}$ axions of two $G^{\alpha}$ multiplets.

\section{B. Embedding into string compactifications}

In this section we try to build explicit models of the KNP and HA mechanisms using the orientifold odd axions of string compactifications. This analysis is a little more involved than the supergravity case since not only do we have to generate a suitable potential for the axions, but we must also stabilize the geometry of the compact space. To achieve this we combine the KKLT or LVS setups with superpotential terms originating from gaugino condensation on D5 branes $[41,50]$,

$W=W_{0}+A e^{-a T}+P e^{-p_{1} G_{1}-p_{2} G_{2}}+Q e^{-q_{1} G_{1}-q_{2} G_{2}}$,

where $T$ can be chosen as needed between the even moduli in each setup.

\section{Inflating in KKLT}

In the simplest case of KKLT moduli stabilization, we consider a single even modulus model, such that the volume of the compact space is given by $\mathcal{V}=t^{3}$, where $t$ is the 2-cycle volume. Allowing for nonzero intersection between the orientifold even and the odd sectors, one can write the Kähler potential in terms of the Kähler coordinates as

$$
\begin{aligned}
K= & -3 \log \left[T+\bar{T}+\frac{k_{1}}{2(S+\bar{S})}\left(G_{1}+\bar{G}_{1}\right)^{2}\right. \\
& \left.+\frac{k_{2}}{2(S+\bar{S})}\left(G_{2}+\bar{G}_{2}\right)^{2}\right]-\log [S+\bar{S}] .
\end{aligned}
$$

The kinetic part of the scalar field Lagrangian then reads

$$
\mathcal{L}_{\text {kin }}=K_{I \bar{J}} \partial_{\mu} \Psi_{I} \partial^{\mu} \Psi_{\bar{J}}=\kappa_{i j} \partial_{\mu} \psi_{i} \partial^{\mu} \psi_{i},
$$

where $\Psi$ denotes the chiral superfield basis, $\Psi=\left\{T, G_{1}, G_{2}, S\right\}$, and $\psi$ the real scalar field basis of moduli space $\psi=\left\{\tau, \rho, b_{1}, c_{1}, b_{2}, c_{2}, s, C_{0}\right\}$. The kinetic matrix for the real degrees of freedom, ignoring the dilaton for the moment, admits the following expansion:

$\kappa_{i j}=\left(\begin{array}{cccccc}\frac{3}{4 \tau^{2}} & 0 & 0 & 0 & 0 & 0 \\ 0 & \frac{3}{4 \tau^{2}} & 0 & 0 & 0 & 0 \\ 0 & 0 & -\frac{3 k_{1}}{4 g_{s} \tau} & 0 & 0 & 0 \\ 0 & 0 & 0 & -\frac{3 k_{2}}{4 g_{s} \tau} & 0 & 0 \\ 0 & 0 & 0 & 0 & -\frac{3 g_{s} k_{1}}{4 \tau} & 0 \\ 0 & 0 & 0 & 0 & 0 & -\frac{3 g_{s} k_{2}}{4 \tau}\end{array}\right)$,

where we keep only the leading terms in $\mathcal{V}$ in each diagonal entry and neglected off-diagonal terms. ${ }^{3}$ We then see that requiring positivity of $\kappa_{i j}$ leads us to consider compactifications with $k_{1}, k_{2}<0$. If these conditions are not met, the $G$ multiplets become ghosts.

The scalar potential resulting from Eqs. (4.7) and (4.8) can be written as $V=V_{0}+V_{1}+V_{2}$, which after minimizing the 4-form axion at $\rho=\frac{-\pi+a\left(b_{1} c_{1} k_{1}+b_{2} c_{2} k_{2}\right)}{a}$ becomes

$$
\begin{aligned}
V_{0}= & \frac{9 W_{0}^{2}\left(b_{1}^{2} k_{1}+b_{2}^{2} k_{2}\right)^{2}}{64 \tau^{5} g_{s}}+A W_{0} e^{-a \tau+\frac{a b_{1}^{2} k_{1}}{2 g_{s}}+\frac{a b_{2}^{2} k_{2}}{2 g_{s}}}\left(-\frac{a g_{s}}{4 \tau^{2}}-\frac{9\left(b_{1}^{2} k_{1}+b_{2}^{2} k_{2}\right)^{2}}{32 \tau^{5} g_{s}}+\frac{3 a\left(b_{1}^{2} k_{1}+b_{2}^{2} k_{2}\right)^{2}}{16 \tau^{4} g_{s}}\right) \\
& +A^{2} e^{-2 a \tau+\frac{a b_{1}^{2} k_{1}}{g_{s}}+\frac{a b_{2}^{2} k_{2}}{g_{s}}}\left(\frac{a^{2} g_{s}}{12 \tau}+\frac{9\left(b_{1}^{2} k_{1}+b_{2}^{2} k_{2}\right)^{2}}{64 \tau^{5} g_{s}}-\frac{3 a\left(b_{1}^{2} k_{1}+b_{2}^{2} k_{2}\right)^{2}}{16 \tau^{4} g_{s}}\right. \\
& \left.+\frac{a^{2}\left(b_{1}^{2} k_{1}+b_{2}^{2} k_{2}\right)^{2}}{16 \tau^{3} g_{s}}-\frac{a\left(-3 g_{s}+a b_{1}^{2} k_{1}+a b_{2}^{2} k_{2}\right)}{12 \tau^{2}}\right),
\end{aligned}
$$

\footnotetext{
${ }^{3}$ The full expression of the kinetic matrix up to order $1 / \mathcal{V}^{2}$ is somewhat complicated and not very illuminating. By presenting only the leading diagonal terms one can understand the behavior of the eigenvalues of $\kappa_{i j}$ in terms of the intersection numbers $k_{1}$ and $k_{2}$ and get a simple estimate of their order of magnitude.
} 


$$
\begin{aligned}
& V_{1}=e^{-\frac{b_{1} p_{1}}{g_{s}}-\frac{b_{2} p_{2}}{g_{s}}} P \cos \left[c_{1} p_{1}+c_{2} p_{2}\right] \times\left[-\frac{a A e^{-a \tau+\frac{a\left(b_{1}^{2} k_{1}+b_{2}^{2} k_{2}\right)}{2 g_{s}}} g_{s}}{4 \tau^{2}}-\frac{9 A e^{-a \tau+\frac{a\left(b_{1}^{2} k_{1}+b_{2}^{2} k_{2}\right)}{2 g_{s}}}\left(b_{1}^{2} k_{1}+b_{2}^{2} k_{2}\right)^{2}}{32 \tau^{5} g_{s}}\right. \\
& +\frac{9 W_{0}\left(b_{1}^{2} k_{1}+b_{2}^{2} k_{2}\right)^{2}}{32 \tau^{5} g_{s}}+\frac{3 a A e^{-a \tau+\frac{a\left(b_{1}^{2} k_{1}+b_{2}^{2} k_{2}\right)}{2 g_{s}}}\left(b_{1}^{2} k_{1}+b_{2}^{2} k_{2}\right)^{2}}{16 \tau^{4} g_{s}}-\frac{a A e^{-a \tau+\frac{a\left(b_{1}^{2} k_{1}+b_{2}^{2} k_{2}\right)}{2 g_{s}}}\left(b_{1} p_{1}+b_{2} p_{2}\right)}{6 \tau^{2}} \\
& -\frac{3 A e^{-a \tau+\frac{a\left(b_{1}^{2} k_{1}+b_{2}^{2} k_{2}\right)}{2 g_{s}}}\left(b_{1}^{2} k_{1}+b_{2}^{2} k_{2}\right)\left(b_{1} p_{1}+b_{2} p_{2}\right)}{8 \tau^{4} g_{s}}+\frac{3 W_{0}\left(b_{1}^{2} k_{1}+b_{2}^{2} k_{2}\right)\left(b_{1} p_{1}+b_{2} p_{2}\right)}{8 \tau^{4} g_{s}} \\
& \left.+\frac{a A e^{-a \tau+\frac{a\left(b_{1}^{2} k_{1}+b_{2}^{2} k_{2}\right)}{2 g_{s}}}\left(b_{1}^{2} k_{1}+b_{2}^{2} k_{2}\right)\left(b_{1} p_{1}+b_{2} p_{2}\right)}{4 \tau^{3} g_{s}}\right]+\left[P \rightarrow Q, p_{1} \rightarrow q_{1}, p_{2} \rightarrow q_{2}\right]
\end{aligned}
$$

and

$$
\begin{aligned}
V_{2} \approx & -P^{2} \frac{\left(k_{2} p_{1}^{2}+k_{1} p_{2}^{2}\right)}{12 \tau^{2} k_{1} k_{2}} e^{\frac{2\left(b_{1} p_{1}+b_{2} p_{2}\right)}{g_{s}}}-Q^{2} \frac{\left(k_{2} q_{1}^{2}+k_{1} q_{2}^{2}\right)}{12 \tau^{2} k_{1} k_{2}} e^{\frac{2\left(b_{1} q_{1}+b_{2} q_{2}\right)}{g_{s}}} \\
& -P Q \frac{\left(k_{2} p_{1} q_{1}+k_{1} p_{2} q_{2}\right)}{6 \tau^{2} k_{1} k_{2}} e^{\frac{b_{1}\left(p_{1}+q_{1}\right)+b_{2}\left(p_{2}+q_{2}\right)}{g_{s}}} \cos \left[c_{1}\left(p_{1}-q_{1}\right)+c_{2}\left(p_{2}-q_{2}\right)\right] .
\end{aligned}
$$

Note that while Eqs. (4.11) and (4.12) are exact, for the sake of short formulas in Eq. (4.13) we have displayed only the first nonvanishing terms in a $1 / \tau$ expansion.

A successful string inflation model must not only give rise to inflation but also be able to keep the noninflationary moduli fixed. In the current context this requires a separation between the physical mass scales of the $\tau, \rho b_{1}$ and $b_{2}$ fields and the $c_{1}$ and $c_{2}$ axions. This scale separation is also a prerequisite for the classical stability of the vacuum: after uplifting the KKLT vacuum of $V_{0}$ is separated from decompactification by a barrier with a height $V_{\text {barrier }} \sim\left|V_{\mathrm{KKLT}}\right| \sim \frac{W_{0}^{2}}{\mathcal{V}^{2}}$. Since any inflationary energy density constitutes an extra form of uplifting, one must have $V_{\text {inf }}<V_{\text {barrier }} \sim \frac{W_{0}^{2}}{\mathcal{V}^{2}}$. The twin requirements of scale separation and vacuum stability therefore impose the following hierarchy on the scalar potential:

$$
V_{0} \gg V_{1}+V_{2}, \quad \text { implying } \quad P, Q \ll \frac{W_{0}}{\sqrt{\tau}} .
$$

Provided this is met one can minimize $V_{0}$ and $V_{1}+V_{2}$ separately, which constitutes a considerable simplification in the search for the $F$-term potential's vacuum.

The leading contribution to the potential, $V_{0}$, depends only on the volume modulus $\tau$ and on the quadratic combination $b_{1}^{2} k_{1}+b_{2}^{2} k_{2}$, and is essentially the generalization of the KKLT potential for compactifications with orientifold odd axions intersecting the volume modulus. Extremizing $V_{0}$ we find that the KKLT minimum is approximately located at $e^{-a \tau+\frac{a\left(b_{1}^{2} k_{1}+b_{2}^{2} k_{2}\right)}{2 g_{s}}} \approx \frac{3 W_{0}}{2 a A \tau}\left(1+\frac{-3+4 a \frac{b_{1}^{2} k 1+b_{2}^{2} k_{2}}{2 g_{s}}}{2 a \tau}\right)$.

As in the supergravity versions of these two axion models, the real partners of the axionic fields are Kähler stabilized at leading order in a circle of fixed radius, determined by the solution to $\frac{\partial V_{0}}{\partial b_{i}}=0,{ }^{4}$ with the angular direction unfixed.

The $V_{1}+V_{2}$ component of the scalar potential depends on both $\tau$ and $b_{1}^{2} k_{1}+b_{2}^{2} k_{2}$ as well as on various linear combinations of $b_{1}$ and $b_{2}$ and on the $c$ axions, in both the KNP and hierarchical axions scenarios, and so $V_{1}+V_{2}$ will lift the remaining flat direction in the $b$ plane. Unfortunately, the structure of the potential complicates the minimization process as soon as one moves away from the (tachyonic) origin of the $b$ plane. This renders our efforts to find analytic expressions for the location of the $\left(b_{1}, b_{2}\right)$ vacuum futile and forces us to resort to numeric methods. In any case, the qualitative picture is identical to that of the supergravity models described in the previous section, and at the end of the process one ends up with all the moduli, volume included, stabilized in a consistent way.

a.KNP alignment mechanism.-At the KKLT minimum, the dominant contribution to $V_{1}$ is

\footnotetext{
${ }^{4}$ Because of the structure of the potential we have not been able to find an analytic expression for the vacuum expectation value of $k_{1} b_{1}^{2}+k_{2} b_{2}^{2}$ that gave a good agreement with the numerical results while still being compact enough to be spelled out here. We therefore proceed with the analysis numerically, keeping in mind that in the cases of interest one finds $\frac{a\left(b_{1}^{2} k_{1}+b_{2}^{2} k_{2}\right)}{2 g} \sim$ $\mathcal{O}(-\mathrm{afew})$, in accordance with the requirement $k_{1}, k_{2}<0$, derived from the absence of ghosts.
} 


$$
\begin{aligned}
V_{1} \approx & -P W_{0} \frac{3 g_{s}-2\left(b_{1} p_{1}+b_{2} p_{2}\right)}{8 \tau^{3}} \\
& \times e^{\frac{b_{1} p_{1}+b_{2} p_{2}}{g_{s}}} \cos \left[c_{1} p_{1}+c_{2} p_{2}\right] \\
& +\left[P \rightarrow Q, p_{1} \rightarrow q_{1}, p_{2} \rightarrow q_{2}\right],
\end{aligned}
$$

which together with the cosine term from $V_{2}$, Eq. (4.13), constitutes the inflationary potential $V_{\text {inf }}$.

Defining the misaligment parameter $\delta$ in terms of the superpotential parameters as

$$
\frac{p_{2}}{p_{1}} \equiv r \quad \text { and } \quad \frac{q_{2}}{q_{1}} \equiv r(1+\delta),
$$

one can map this stringy model onto the field theory analysis of Sec. II. The potential for the canonically normalized $c$ axions

$$
\phi_{1}=\tilde{f}_{1} c_{1} \quad \text { and } \quad \phi_{2}=\tilde{f}_{2} c_{2}
$$

can then be written, upon uplifting, as

$$
\begin{aligned}
V_{\mathrm{inf}}= & \Lambda_{1}^{4}\left(1-\cos \left[\frac{\phi_{1}}{\tilde{f}_{1} / p_{1}}+\frac{\phi_{2}}{\tilde{f}_{2} /\left(p_{1} r\right)}\right]\right)+\Lambda_{2}^{4}\left(1-\cos \left[\frac{\phi_{1}}{\tilde{f}_{1} / q_{1}}+\frac{\phi_{2}}{\tilde{f}_{2} /\left(q_{1} r(1+\delta)\right)}\right]\right) \\
& +\Lambda_{3}^{4}\left(-1 \cos \left[\frac{\phi_{1}}{\tilde{f}_{1} /\left(p_{1}-q_{1}\right)}+\frac{\phi_{2}}{\tilde{f}_{2} /\left(r p_{1}-(1+\delta) r q_{1}\right)}\right]\right),
\end{aligned}
$$

where $\Lambda_{1}, \Lambda_{3}$, and $\Lambda_{3}$ can be read off Eqs. (4.13) and (4.16) and therefore scale as

$\Lambda_{1}^{4} \sim \mathcal{O}\left(\frac{W_{0} P}{\tau^{3}}\right), \quad \Lambda_{2}^{4} \sim \mathcal{O}\left(\frac{W_{0} Q}{\tau^{3}}\right), \quad \Lambda_{3}^{4} \sim \mathcal{O}\left(\frac{P Q}{\tau^{2}}\right)$

From Eq. (4.10) one also defines

$$
\tilde{f}_{1,2}=\sqrt{\frac{-3 k_{1,2} g_{s}}{2 \tau}}
$$

and so one finds that the large effective decay constant is then written in terms of the compactification parameters as

$$
f_{\text {eff }}=\frac{\sqrt{\tilde{f}_{2}^{2}+r^{2} \tilde{f}_{1}^{2}}}{r q_{1} \delta} .
$$

If there are no large asymmetries either in the intersection numbers, $k_{1} \sim k_{2}$, or in the superpotential exponents, $r \sim 1$, one finds $f_{\text {eff }} \sim \frac{\tilde{f}_{1}}{q_{1} \delta}$. Working in a region of parameter space where $k_{1} \sim k_{2} \sim-\mathcal{O}(1), g_{s} \ll 1$, and $\tau \sim \mathcal{O}(10)$, ${ }^{5}$ one tends to find $\tilde{f}_{1,2} \lesssim \mathcal{O}(0.1)$. Furthermore noting that since the $G$ moduli potential generated by gaugino condensation on D5-branes implies $q_{1}=\frac{2 \pi}{N}$, one sees that for $f_{\text {eff }} \gtrsim 5 M_{P}$ one must have the misalignment parameter tuned at the level of

\footnotetext{
${ }^{5} \mathrm{As}$ is often the case in models of string inflation, the compactification volume is closely tied with the scale of inflation, which is in turn subject to observational bounds on the amplitude of the curvature perturbations. We therefore expect models with significantly larger $\tau$ to be at least extremely contrived, if not inconsistent with observations.
}

$$
\delta \lesssim \mathcal{O}\left(\frac{N}{300}\right)
$$

There are several possible avenues to arrange for tuning the alignment parameter into a range of $\delta=0.01 \ldots 0.1$. One possibility is to employ CY manifolds which contain nontrivial linear combinations of the symplectic-basis 2-cycles (Poincaré dual to so-called "partially ample" 4cycle divisors). The gauge kinetic function of the 5branes wrapping these linear-combination 2-cycles then depends on a linear combination $k_{1} G_{1}+k_{2} G_{2}$ with $k_{1}, k_{2}$ numbers which are related to the intersection numbers determining the nontrivial linear-combination 2-cycle. By sampling over such 2-cycles and CYs containing these types of linear-combination 2-cycles, we should be able to find examples where $k_{2}=k_{1}(1+\mathcal{O}(\delta))$, providing the necessary level of alignment.

Alternatively, we may use D7-brane gaugino condensates (or ED3-ED1 instantons) instead of D5-branes, and then turn magnetic $F_{2}$ flux on them [34]. The D7-gauge kinetic function will then be $f_{a, D 7}=T_{a}+f^{i} G_{i}+\cdots$ with $f^{i}$ being the quanta of $F_{2}$-flux possible on the 4cycle of the D7 stack wraps, and $G_{i}$ those $G$-axion fields which appear in the D7-Chern-Simons action once the $f^{i}$ are turned on. In this case, tuning the magnetic flux quanta may allow for reaching the necessary alignment $f^{2}=f^{1}(1+\mathcal{O}(\delta))$. We note further that the $\exp (-a T)$ prefactors present in the nonperturbative terms from the D7-brane stacks or ED3-ED1s contribute part of the tuning of the effective instanton prefactors $P, Q$ similar to what we find in Sec. IV C for LVS. In that case we expect values of the residual effective $P, Q \sim$ $\mathcal{O}(0.01 \cdots 0.1)$.

Finally, in Tables I and II we present two numerical examples of the model described above. 
TABLE I. Input parameters for the string embedding of the KNP mechanism.

\begin{tabular}{lcccccccccccc}
\hline \hline & $W_{0}$ & $A$ & $a$ & $P$ & $Q$ & $p_{1}$ & $q_{1}$ & $r$ & $\delta$ & $k_{1}$ & $k_{2}$ \\
\hline $\mathrm{KNP}_{1}$ & 0.11 & 5 & 0.1 & $10^{-4}$ & $10^{-4}$ & $\pi / 2$ & $\pi / 2$ & 1 & 0.02 & -3 & -3 & 0.5 \\
$\mathrm{KNP}_{2}$ & 0.099 & 1 & 0.1 & $10^{-4}$ & $10^{-3}$ & $\pi / 5$ & $\pi / 5$ & 1.5 & 0.1 & -7 & -7 & 0.3 \\
\hline \hline
\end{tabular}

TABLE II. Compactification features and inflationary parameters. All dimensionful quantities expressed in Planckian units. We note that while we are presenting here only one minimum for $\left(b_{1}, b_{2}\right)$, there are in general two degenerate minima.

\begin{tabular}{lcccccr}
\hline \hline & $\tau$ & $b_{1}$ & $b_{2}$ & $\Lambda_{1}^{4} \times 10^{12}$ & $\Lambda_{2}^{4} \times 10^{12}$ & $\Lambda_{3}^{4} \times 10^{12}$ \\
\hline $\mathrm{KNP}_{1}$ & 51 & 0.59 & -0.59 & 11 & 11 & 0.98 \\
$\mathrm{KNP}_{2}$ & 34 & 0.29 & -0.18 & 18 & 180 & 9.4 \\
\hline \hline
\end{tabular}

TABLE III. Input parameters for the string embedding of the hierarchical axions' mechanism.

\begin{tabular}{|c|c|c|c|c|c|c|c|c|c|c|c|}
\hline & $W_{0}$ & $A$ & $a$ & $X_{1}$ & $X_{2}$ & $p_{1}$ & $q_{1}$ & $q_{2}$ & $k_{1}$ & $k_{2}$ & $g_{s}$ \\
\hline $\mathrm{HA}_{1}$ & 0.022 & 1 & 0.1 & 0.0001 & 0.0001 & $\pi / 10$ & $\pi$ & $\pi / 10$ & -7 & -7 & 0.4 \\
\hline $\mathrm{HA}_{2}$ & 0.0099 & 1 & 0.2 & 0.0001 & 0.0001 & $\pi / 7$ & $2 \pi$ & $\pi / 7$ & -2 & -2 & 0.4 \\
\hline
\end{tabular}

b.Hierarchical axions mechanism.-The analysis of the inflationary potential of Eq. (4.40) proceeds in a similar manner to the closely related KNP mechanism. By making the discrete choice $p_{2}=0$ and working in the limit $q_{1} \gg p_{1}, q_{2}$, we find

$$
\begin{aligned}
V_{1}+V_{2}= & -P W_{0} \frac{3 g_{s}-2 b_{1} p_{1}}{8 \tau^{3}} e^{\frac{b_{1} p_{1}}{g_{s}}} \cos \left[c_{1} p_{1}\right]-Q W_{0} \frac{3 g_{s}-2\left(b_{1} q_{1}+b_{2} q_{2}\right)}{8 \tau^{3}} e^{\frac{b_{1} q_{1}+b_{2} q_{2}}{g_{s}}} \cos \left[c_{1} q_{1}+c_{2} q_{2}\right] \\
& -P Q \frac{p_{1} q_{1}}{6 \tau^{2} k_{1}} e^{\frac{b_{1} p_{1}}{g_{s}}+\frac{b_{1} q_{1}}{g_{s}}+\frac{b_{2} q_{2}}{g_{s}}} \cos \left[c_{1} p_{1}-c_{1} q_{1}-c_{2} q_{2}\right]-P^{2} \frac{p_{1}^{2}}{12 \tau^{2} k_{1}} e^{\frac{2 b_{1} p_{1}}{g_{s}}}-Q^{2} \frac{k_{2} q_{1}^{2}+k_{1} q_{2}^{2}}{12 \tau^{2} k_{1} k_{2}} e^{\frac{2 b_{1} q_{1}}{g_{s}}+\frac{2 b_{2} q_{2}}{g_{s}}} .
\end{aligned}
$$

As before, $V_{1}+V_{2}$ is simultaneously responsible for the stabilization of the linear combination ${ }^{6} b_{1} p_{1}+b_{2} p_{2}$ and for the inflationary dynamics. After integrating out the $b$ fields and uplifting it reduces to

$$
V_{\mathrm{inf}}=\Lambda_{1}^{4}\left(1-\cos \left[p_{1} c_{1}\right]\right)+\Lambda_{2}^{4}\left(1-\cos \left[q_{1} c_{1}+q_{2} c_{2}\right]\right)+\Lambda_{3}^{4}\left(1-\cos \left[\left(p_{1}-q_{1}\right) c_{1}+q_{2} c_{2}\right]\right),
$$

where the leading scaling of the amplitudes is given by

$$
\Lambda_{1}^{4} \sim \mathcal{O}\left(\frac{P W_{0}}{\tau^{3}}\right), \quad \Lambda_{2}^{4} \sim \mathcal{O}\left(\frac{Q W_{0}}{\tau^{3}}\right), \quad \Lambda_{3}^{4} \sim \mathcal{O}\left(\frac{P Q}{\tau^{2}}\right) .
$$

Noting that to leading order $p_{1}-q_{1} \sim-q_{1}$ the potential can be reduced to the simple form of (2.1) where the amplitude of the mixed cosine is now $\Lambda_{2}^{4}+\Lambda_{3}^{4}$. Writing the potential in terms of the canonically normalized fields, defined by Eq. (4.18), one finds

$$
V_{\mathrm{inf}} \approx \Lambda_{1}^{4}\left(1-\cos \left[\frac{p_{1}}{\tilde{f}_{1}} \phi_{1}\right]\right)+\left(\Lambda_{2}^{4}+\Lambda_{3}^{4}\right)\left(1-\cos \left[\frac{q_{1}}{\tilde{f}_{1}} \phi_{1}+\frac{q_{2}}{\tilde{f}_{2}} \phi_{2}\right]\right)
$$

implying that the large effective decay constant is

$$
f_{\text {eff }}=\tilde{f}_{2} \frac{q_{1}}{p_{1} q_{2}} .
$$

In Tables III and IV we present a couple of numerical examples.

\footnotetext{
${ }^{6} \mathrm{We}$ do not present an explicit analytic formula for the location of this minimum as it involves solving a nonalgebraic equation.
} 
TABLE IV. Compactification features and inflationary parameters. All dimensionful quantities expressed in Planckian units. We note that while we are presenting here only one minimum for $\left(b_{1}, b_{2}\right)$, in general there are two degenerate minima.

\begin{tabular}{cccccccc}
\hline \hline & $\tau$ & $b_{1}$ & $b_{2}$ & $\Lambda_{1}^{4} \times 10^{12}$ & $\Lambda_{2}^{4} \times 10^{12}$ & $\Lambda_{3}^{4} \times 10^{12}$ & $f_{\text {eff }}$ \\
\hline $\mathrm{HA}_{1}$ & 50 & 0.01 & -0.49 & 1.7 & 1.6 & 0.06 & 9.1 \\
$\mathrm{HA}_{2}$ & 30 & -0.013 & -0.71 & 4 & 2.7 & 0.9 & 6.2 \\
\hline \hline
\end{tabular}

\section{Inflating in LVS}

Having shown that effective trans-Planckian decay constants can coexist with stabilized moduli in the context of a single modulus KKLT compactification we now set out to do the same within the LVS of type IIB flux compactifications.

Our starting point is a compactification which beyond the two orientifold odd $G$-fields giving rise to inflation also includes a pair of orientifold even $T$ moduli, whose triple intersection numbers lead to a Swiss-cheese geometry. This setup implies the following Kähler potential:

$$
K=-2 \log \left[\Sigma_{1}^{3 / 2}-\Sigma_{2}^{3 / 2}+\xi(S+\bar{S})^{3 / 2}\right]-\log [S+\bar{S}],
$$

where

$$
\begin{aligned}
& \Sigma_{1} \equiv T_{1}+\bar{T}_{1}+\frac{k_{11}}{2(S+\bar{S})}\left(G_{1}+\bar{G}_{1}\right)^{2}+\frac{k_{12}}{2(S+\bar{S})}\left(G_{2}+\bar{G}_{2}\right)^{2}, \\
& \Sigma_{2} \equiv T_{2}+\bar{T}_{2}+\frac{k_{21}}{2(S+\bar{S})}\left(G_{1}+\bar{G}_{1}\right)^{2}+\frac{k_{22}}{2(S+\bar{S})}\left(G_{2}+\bar{G}_{2}\right)^{2} .
\end{aligned}
$$

Given that we want to stabilize the moduli à la LVS we have also included in $K$ the $\mathcal{O}\left(\alpha^{\prime 3}\right)$ correction originating from the fourth order curvature correction to the ten-dimensional action.

Before we analyze the $F$-term potential that follows from Eqs. (4.7) and (4.29), we compute the kinetic matrix for the real degrees of freedom $\psi=\left\{\tau_{1}, \rho_{1}, \tau_{2}, \rho_{2}, b_{1}, b_{2}, c_{1}, c_{2}\right\}$, finding

$$
\kappa_{i j}=\left(\begin{array}{cccc|cccc}
\frac{3}{4 \tau_{1}^{2}} & 0 & 0 & 0 & 0 & 0 & 0 & 0 \\
0 & \frac{3}{4 \tau_{1}^{2}} & 0 & 0 & -\frac{3 c_{1} k_{11}}{2 \tau_{1}^{2}} & -\frac{3 c_{2} k_{12}}{2 \tau_{1}^{2}} & 0 & 0 \\
0 & 0 & \frac{3}{8 \tau_{1}^{3 / 2} \sqrt{\tau_{2}}} & 0 & 0 & 0 & 0 & 0 \\
0 & 0 & 0 & \frac{3}{8 \tau_{1}^{3 / 2} \sqrt{\tau_{2}}} & -\frac{3 c_{1} k_{21}}{4 \tau_{1}^{3 / 2} \sqrt{\tau_{2}}} & -\frac{3 c_{2} k_{22}}{4 \tau_{1}^{3 / 2} \sqrt{\tau_{2}}} & 0 & 0 \\
\hline 0 & -\frac{3 c_{1} k_{11}}{2 \tau_{1}^{2}} & 0 & -\frac{3 c_{1} k_{21}}{4 \tau^{3 / 2} \sqrt{\tau_{2}}} & & & \\
0 & -\frac{3 c_{2} k_{12}}{2 \tau_{1}^{2}} & 0 & -\frac{3 c_{22}}{4 \tau_{1}^{3 / 2} \sqrt{\tau_{2}}} & 0 & \tilde{\kappa}_{m n} & \\
0 & 0 & 0 & 0 & & & \\
0 & 0 & 0 & 0 & & &
\end{array}\right)
$$

with

$$
\tilde{\kappa}_{m n}=\left(\begin{array}{cccc}
-\frac{3 k_{11}}{4 g_{s} \tau_{1}}+\frac{3 c_{1}^{2} k_{21}^{2}}{8 \tau_{1}^{3 / 2} \sqrt{\tau_{2}}}+\frac{3 k_{21} \sqrt{\tau_{2}}}{4 g_{s} \tau_{1}^{3 / 2}} & \frac{3 c_{1} c_{2} k_{21} k_{22}}{4 \tau_{1}^{3 / 2} \sqrt{\tau_{2}}} & 0 & 0 \\
\frac{3 c_{1} c_{2} k_{21} k_{22}}{4 \tau_{1}^{3 / 2} \sqrt{\tau_{2}}} & -\frac{3 k_{12}}{4 g_{s} \tau_{1}}+\frac{3 c_{3}^{2} k_{22}^{2}}{8 \tau_{1}^{3 / 2} \sqrt{\tau_{2}}}+\frac{3 k_{22} \sqrt{\tau_{2}}}{4 g_{s} \tau_{1}^{3 / 2}} & 0 & 0 \\
0 & 0 & -\frac{3 g_{s} k_{11}}{4 \tau_{1}}+\frac{3 g_{s} k_{21} \sqrt{\tau_{2}}}{4 \tau_{1}^{3 / 2}} & 0 \\
0 & 0 & 0 & -\frac{3 g_{s} k_{12}}{4 \tau_{1}}+\frac{3 g_{s} k_{22} \sqrt{\tau_{2}}}{4 \tau_{1}^{3 / 2}}
\end{array}\right),
$$

where we have set, in anticipation of what is to come, $b_{1}=b_{2}=0$. Noting that the eigenvalues of $\kappa_{i j}$ are mostly determined by the diagonal entries, we see from Eq. (4.32) that for the orientifold odd moduli to have positive kinetic terms one must arrange

$$
k_{11} \sqrt{\tau_{1}}-k_{21} \sqrt{\tau_{2}}<0 \quad \text { and } \quad k_{12} \sqrt{\tau_{1}}-k_{22} \sqrt{\tau_{2}}<0 .
$$

The scalar potential that generalizes LVS for $k_{+--} \neq 0$ takes the form 


$$
\begin{aligned}
V_{\mathrm{LVS}} & =\frac{9\left(b_{1}^{2} k_{11}+b_{2}^{2} k_{12}\right)^{2} W_{0}^{2}}{64 g_{s} \tau_{1}^{5}}+\frac{3 \xi W_{0}^{2}}{32 \sqrt{g_{s}} \tau_{1}^{9 / 2}}-\frac{a^{2} A^{2}\left(b_{1}^{2} k_{12} k_{21}^{2}+b_{2}^{2} k_{11} k_{22}^{2}\right)}{12 k_{11} k_{12} \tau_{1}^{2}} e^{a\left(\frac{b^{2} k_{21}+b_{2}^{2} k_{22}}{g_{s}}-2 \tau_{2}\right)} \\
& +\frac{a^{2} A^{2} g_{s} \sqrt{\tau_{2}}}{6 \tau_{1}^{3 / 2}} e^{a\left(\frac{b_{1}^{2} k_{21}+b_{2}^{2} k_{22}}{g_{s}}-2 \tau_{2}\right)}-\frac{g_{s} a A W_{0} \tau_{2}}{4 \tau_{1}^{3}} e^{a\left(\frac{b_{1}^{2} k_{21}+b_{2}^{2} k_{22}}{2 g_{s}}-\tau_{2}\right)} .
\end{aligned}
$$

Solving $\partial V_{\mathrm{LVS}} / \partial b_{1}=0, \partial V_{\mathrm{LVS}} / \partial b_{2}=0$ one finds that the origin of the $b$ plane $\left(b_{1}, b_{2}\right)=(0,0)$ is an extremum of $V_{\text {LVS }}$. At this point in moduli space, the even moduli vacuum is determined in the usual way to lie at

$\tau_{1}^{3 / 2}=\frac{3 W_{0} \sqrt{\tau_{2}}\left(-1+a \tau_{2}\right)}{a A\left(-1+4 a \tau_{2}\right)} e^{a \tau_{2}} \sim \frac{3 W_{0 \sqrt{\tau_{2}}}}{4 a A} e^{a \tau_{2}}\left(1-\frac{3}{4 a \tau_{2}}\right)$,

$\tau_{2}^{3 / 2}=\frac{\xi\left(-1+4 a \tau_{2}\right)^{2}}{16 g_{s}^{3 / 2} a \tau_{2}\left(-1+a \tau_{2}\right)} \sim \frac{\xi}{g_{s}^{3 / 2}}\left(1+\frac{1}{2 a \tau_{2}}\right)$.

This allows us to show the second derivatives of $V_{\text {LVS }}$ at the origin of the $b$ plane are given by

$$
\begin{aligned}
& \frac{\partial^{2} V_{\mathrm{LVS}}}{\partial b_{1}^{2}}=-\frac{9 \xi^{1 / 3} k_{21} W_{0}^{2}}{64 \sqrt{g_{s}} \tau_{1}^{9 / 2}}-\frac{3 \xi^{2 / 3} k_{21}^{2} W_{0}^{2}}{32 g_{s} k_{11} \tau_{1}^{5}}, \\
& \frac{\partial^{2} V_{\mathrm{LVS}}}{\partial b_{1} \partial b_{2}}=0, \\
& \frac{\partial^{2} V_{\mathrm{LVS}}}{\partial b_{2}^{2}}=-\frac{9 \xi^{1 / 3} k_{22} W_{0}^{2}}{64 \sqrt{g_{s}} \tau_{1}^{9 / 2}}-\frac{3 \xi^{2 / 3} k_{22}^{2} W_{0}^{2}}{32 g_{s} k_{12} \tau_{1}^{5}} .
\end{aligned}
$$

We can see that the more natural scenario involves having all $k<0$, such that $\frac{\partial^{2} V_{\text {LVs }}}{\partial b_{i} \partial b_{j}} \geq 0$. In such a case, ghosts are avoided if the magnitudes of the intersection numbers obey

$$
\left|k_{11}\right|>\left|k_{21}\right| \sqrt{\tau_{2} / \tau_{1}}, \quad\left|k_{12}\right|>\left|k_{22}\right| \sqrt{\tau_{2} / \tau_{1}},
$$

as follows from Eq. (4.33). If these conditions are met, the $b$ moduli are consistently stabilized by a quadratic potential at the origin, considerably simplifying the analysis of the inflationary potential.

\section{D5 generated potential}

The full scalar potential following from Eqs. (4.7) and (4.29) has the following structure:

$$
V=V_{\mathrm{LVS}}+V_{1}+V_{2}
$$

where the D5 generated contributions take the form

$$
\begin{aligned}
V_{1}= & \frac{3 P W_{0} \xi}{64 a \sqrt{g_{s}} \tau_{1}^{9 / 2} \tau_{2}} \cos \left[c_{1} p_{1}+c_{2} p_{2}\right] \\
& +\frac{3 Q W_{0} \xi}{64 a \sqrt{g_{s}} \tau_{1}^{9 / 2} \tau_{2}} \cos \left[c_{1} q_{1}+c_{2} q_{2}\right]
\end{aligned}
$$

and

$$
\begin{aligned}
V_{2}= & -\frac{P Q}{\tau_{1}^{2}} \frac{\left(k_{12} p_{1} q_{1}+k_{11} p_{2} q_{2}\right)}{6 k_{11} k_{12}} \\
& \times \cos \left[c_{1} p_{1}+c_{2} p_{2}-c_{1} q_{1}-c_{2} q_{2}\right] \\
& -\frac{P^{2}}{\tau_{1}^{2}} \frac{\left(k_{12} p_{1}^{2}+k_{11} p_{2}^{2}\right)}{12 k_{11} k_{12}}-\frac{Q^{2}}{\tau_{1}^{2}} \frac{\left(k_{12} q_{1}^{2}+k_{11} q_{2}^{2}\right)}{12 k_{11} k_{12}} .
\end{aligned}
$$

From Eqs. (4.34), (4.40), and (4.41) one can read off the volume scaling of each component of $V$ close to the LVS minimum,

$$
\begin{aligned}
V_{\mathrm{LVS}} & \sim \mathcal{O}\left(-\frac{W_{0}^{2}}{\mathcal{V}^{3} \log \mathcal{V}}\right), \quad V_{1} \sim \mathcal{O}\left(\frac{Q W_{0}+P W_{0}}{\mathcal{V}^{3} \log \mathcal{V}}\right) \\
V_{2} & \sim \mathcal{O}\left(\frac{P Q+P^{2}+Q^{2}}{\mathcal{V}^{4 / 3}}\right) .
\end{aligned}
$$

A feature of LVS moduli stabilization is that the resulting minimum is nonsupersymmetric and AdS. Upon uplifting, the barrier separating the LVS vacuum from decompactification has a height roughly equal to the depth of the original AdS minimum: $V_{\text {barrier }} \sim \mathcal{O}\left(-\frac{W_{0}^{2}}{\mathcal{V}^{3} \log \mathcal{V}}\right)$. Since any sort of inflationary energy density will contribute to the $F$ term potential as an additional uplift term (see [51] for a discussion), classical stability of the LVS vacuum requires

$$
V_{\text {barrier }} \sim-V_{\text {LVS }} \gg V_{\text {inf }}=V_{1}+V_{2} \text {. }
$$

This can be seen as a constraint on the magnitude of the D5 brane generated $P$ and $Q$ parameters,

$$
P, Q \ll \frac{W_{0}}{\mathcal{V}^{5 / 6} \sqrt{\log \mathcal{V}}}
$$

With this hierarchy not only do we ensure the classical stability of the vacuum but we also make it possible to decouple the light $c$ axions from their heavier $b$ partners and from the orientifold even moduli. 
TABLE V. Input parameters for the LVS string embedding.

\begin{tabular}{|c|c|c|c|c|c|c|c|c|c|c|c|c|c|c|c|}
\hline & $W_{0}$ & $A$ & $a$ & $P$ & $Q$ & $p_{1}$ & $q_{1}$ & $r$ & $\delta$ & $k_{11}$ & $k_{12}$ & $k_{21}$ & $k_{22}$ & $g_{s}$ & $\xi$ \\
\hline $\mathrm{KNP}_{1}$ & 1 & 1 & $\pi$ & $10^{-4}$ & $10^{-3}$ & $\frac{\pi}{2}$ & $\frac{\pi}{2}$ & 1 & 0.01 & -3 & -3 & -3 & -3 & 0.3 & 0.5 \\
\hline $\mathrm{KNP}_{2}$ & 8 & 2 & $\frac{2 \pi}{5}$ & 0.01 & 0.01 & $\frac{\pi}{5}$ & $\frac{\pi}{5}$ & 0.8 & 0.1 & -6 & -6 & -6 & -6 & 0.3 & 1 \\
\hline
\end{tabular}

TABLE VI. Input parameters for the LVS string embedding.

\begin{tabular}{|c|c|c|c|c|c|c|c|c|c|c|c|c|c|c|c|}
\hline & $W_{0}$ & $A$ & $a$ & $P$ & $Q$ & $p_{1}$ & $p_{2}$ & $q_{1}$ & $q_{2}$ & $k_{11}$ & $k_{12}$ & $k_{21}$ & $k_{22}$ & $g_{s}$ & $\xi$ \\
\hline $\mathrm{HA}_{1}$ & 1 & 1 & $\frac{2 \pi}{5}$ & $10^{-3}$ & $10^{-3}$ & 0 & $\frac{\pi}{11}$ & $\frac{\pi}{11}$ & $\pi$ & -1 & -1 & -1 & -1 & 0.4 & 1.5 \\
\hline $\mathrm{HA}_{2}$ & 10 & 2 & $\pi$ & $5 \times 10^{-3}$ & $5 \times 10^{-3}$ & 0 & $\frac{2 \pi}{15}$ & $\frac{2 \pi}{25}$ & $\pi$ & -10 & -10 & -1 & -1 & 0.5 & 0.7 \\
\hline
\end{tabular}

The inflationary part of the potential, $V_{\text {inf }}=V_{1}+V_{2}$, can be written in the form of Eq. (3.10) with the identifications

$\Lambda_{1}^{4} \equiv \frac{3 P W_{0} \xi}{64 a \sqrt{g_{s}} \tau_{1}^{9 / 2} \tau_{2}}, \quad \Lambda_{2}^{4} \equiv \frac{3 Q W_{0} \xi}{64 a \sqrt{g_{s}} \tau_{1}^{9 / 2} \tau_{2}}$

$\Lambda_{3}^{4} \equiv-\frac{P Q}{\tau_{1}^{2}} \frac{\left(k_{12} p_{1} q_{1}+k_{11} p_{2} q_{2}\right)}{6 k_{11} k_{12}}$

and

$$
\begin{aligned}
& \tilde{f}_{1} \equiv\left(-\frac{3 g_{s} k_{11}}{2 \tau_{1}}+\frac{3 g_{s} k_{21} \sqrt{\tau_{2}}}{2 \tau_{1}^{3 / 2}}\right)^{1 / 2}, \\
& \tilde{f}_{2} \equiv\left(-\frac{3 g_{s} k_{12}}{2 \tau_{1}}+\frac{3 g_{s} k_{22} \sqrt{\tau_{2}}}{2 \tau_{1}^{3 / 2}}\right)^{1 / 2} .
\end{aligned}
$$

Because of the fact that the $b$ moduli are stabilized at the origin by $V_{\text {LVS }}$, which is independent of whether we want to inflate via the KNP or the HA mechanisms, the above analysis applies to both cases.

In Tables $\mathrm{V}$ and VI we present the input parameters for the KNP and HA numerical examples, whose compactification and inflationary parameters are displayed in Table VII. Semirealistic models require two distinct tunings, as mentioned above. One starts by choosing the superpotential exponents and the intersection numbers such that the largest effective decay constant is $\geq 5 M_{P}$. Then one chooses the stabilized volume and the $P$ and $Q$ parameters to set the scales in the inflationary potential. While doing

TABLE VII. Compactification features and inflationary parameters. Dimensionful quantities in units of $M_{P}$.

\begin{tabular}{lcccccc}
\hline \hline & $\tau_{1}$ & $\tau_{2}$ & $\Lambda_{1}^{4} \times 10^{12}$ & $\Lambda_{2}^{4} \times 10^{12}$ & $\Lambda_{3}^{4} \times 10^{12}$ & $f_{\text {eff }}$ \\
\hline $\mathrm{KNP}_{1}$ & 48.2 & 2.22 & 0.016 & 0.16 & 11.8 & 13.4 \\
$\mathrm{KNP}_{2}$ & 51.3 & 3.64 & 30 & 30 & 709 & 5.0 \\
$\mathrm{HA}_{1}$ & 19.3 & 3.59 & 41 & 41 & 443 & 5.1 \\
$\mathrm{HA}_{2}$ & 42.3 & 1.70 & 21 & 21 & 306 & 11.23 \\
\hline \hline
\end{tabular}

this one must ensure stability of the LVS vacuum, which requires tuning $P$ and $Q$ small as in Eq. (4.44). In these models tuning the gauge groups ranks large and $P$ and $Q$ small seems unavoidable.

\section{E. ED3/ED1 generated potential}

An interesting modification to the scenario analyzed above is to consider that the inflationary potential is generated by ED3/ED1 terms in the superpotential rather than by gaugino condensation on D5 branes. In this case the superpotential is

$$
W=W_{0}+A e^{-a T_{2}}\left(1+P e^{-p_{1} G_{1}-p_{2} G_{2}}+Q e^{-q_{1} G_{1}-q_{2} G_{2}}\right) .
$$

The benefit of this setup is that the less volume suppressed part of the inflationary potential is now down by an extra power of the compactification volume, which alleviates the tuning in the $P$ and $Q$ coefficients. This can be seen by comparing Eqs. (4.7) and (4.47), noting that the odd axion part of $W$ receives an extra $e^{-a T_{2}} \sim \frac{1}{\mathcal{V}}$ suppression.

In the limit in which one can decouple the $c$ axions from the remaining heavy fields, the minimization of the orientifold even and $b$ moduli carries over from the previous section, with the vacuum defined by $b_{1}=b_{2}=$ 0 and by Eqs. (4.35) and (4.36). The dominant contributions to the $c$ axion potential now originate from terms of the form $\left(\frac{\partial W}{\partial T_{2}}\right)^{2}+W_{0} \frac{\partial W}{\partial T_{2}}$, whereas in the D5 case they came from $\left(\frac{\partial W}{\partial G_{i}}\right)^{2}+W_{0} \frac{\partial W}{\partial G_{i}}$. This is the reason why taking Eq. (4.47) instead of (4.7) does not simply imply an extra overall $1 / \mathcal{V}$ suppression. Explicit computation of the potential yields $V=V_{\mathrm{LVS}}+V_{1}+V_{2}$ with

$$
\begin{aligned}
V_{1}= & -\frac{9 P \xi W_{0}^{2}}{64 a \sqrt{g_{s}} \tau_{1}^{9 / 2} \tau_{2}} \cos \left[c_{1} p_{1}+c_{2} p_{2}\right] \\
& -\frac{9 Q \xi W_{0}^{2}}{64 a \sqrt{g_{s}} \tau_{1}^{9 / 2} \tau_{2}} \cos \left[c_{1} q_{1}+c_{2} q_{2}\right]
\end{aligned}
$$


and

$$
\begin{aligned}
V_{2}= & \frac{3\left(P^{2}+Q^{2}\right) \xi W_{0}^{2}}{32 \sqrt{g_{s}} \tau_{1}^{9 / 2}} \\
& +\frac{3 P Q \xi W_{0}^{2}}{16 \sqrt{g_{s}} \tau_{1}^{9 / 2}} \cos \left[c_{1} p_{1}+c_{2} p_{2}-c_{1} q_{1}-c_{2} q_{2}\right],
\end{aligned}
$$

from which we can read the leading volume scaling

$$
\begin{aligned}
V_{\mathrm{LVS}} & \sim \mathcal{O}\left(-\frac{W_{0}^{2}}{\mathcal{V}^{3} \log \mathcal{V}}\right), \quad V_{1} \sim \mathcal{O}\left(\frac{Q W_{0}^{2}+P W_{0}^{2}}{\mathcal{V}^{3} \log \mathcal{V}}\right), \\
V_{2} & \sim \mathcal{O}\left(\frac{W_{0}^{2}\left(P Q+P^{2}+Q^{2}\right)}{\mathcal{V}^{3}}\right) .
\end{aligned}
$$

As in the D5 case, vacuum stability requires $V_{1}, V_{2} \ll$ $V_{\text {LVS }}$, which now implies

$$
P, Q \ll \frac{1}{\sqrt{\log \mathcal{V}}},
$$

constituting a less severe bound than that of Eq. (4.44).

The tuning required to obtain trans-Planckian decay constants is exactly as before; apart from the different volume scaling, Eqs. (4.48) and (4.49) are identical to (4.40) and (4.41), respectively.

To illustrate the advantages of this setup consider the first numerical example of the KNP mechanism presented in the previous section: $\mathrm{KNP}_{1}$ in Table $\mathrm{V}$. To ensure vacuum stability we have required that $\left|V_{\mathrm{LVS}}\right| \geq 10\left|V_{1}+V_{2}\right|$, and this in turn required $(P, Q)=\left(10^{-4}, 10^{-3}\right)$. Taking the same criterion for vacuum stability, with the ED3/ED1 generated potential one gets instead $(P, Q)=$ $\left(10^{-2}, 3 \times 10^{-2}\right)$, a considerable decrease in the level of fine-tuning in the model. A similar exercise reveals that one gains a factor of $\mathcal{O}(10-100)$ in the tuning of $P$ and $Q$ in the remaining examples of Sec. IV D. ${ }^{7}$

\section{DISCUSSION}

Reliable string theoretic large field models of inflation have been sought for a long time, especially if the BICEP2 result holds. Natural inflation is an elegant model of inflation due to the shift symmetry which protects its potential from dangerous corrections. It also exhibits an

\footnotetext{
${ }^{7}$ Here we are comparing different realizations of inflation at constant volume. By keeping $\mathcal{V}$ constant and changing $P$ and $Q$ we are increasing the scales of $V_{1}$ and $V_{2}$. Realistically these scales are to be set by the normalization of curvature perturbations once we fix the large effective decay constant. We therefore expect that everything else being equal, by taking ED3/ED1 one can get inflation at the right scale for compactifications with larger $P$ and $Q$ as well as larger $\mathcal{V}$.
}

interesting range of predictions for the spectral index $n_{s}$ and the tensor-to-scalar ratio $r$ which fits current data. The main challenge of the model is therefore a plausible embedding in a fundamental theory which will explain the decay constant $f \geq 5 M_{p}$, necessary to match observations. In string theory one expects many axions, making natural inflation seemingly natural. Nevertheless, there are two notable obstacles. First, as we explained in the Introduction, in string theory the decay constants tend to be parametrically smaller than $M_{p}$. Second, these axions are intricately coupled to moduli fields. Hence, as we explained, one must stabilize all moduli and make sure that they do not spoil slow-roll inflation, and that inflation does not destabilize them. Previous works $[27,32,33]$ demonstrated that two fundamental axions produce such an effective decay constant on the field theory level, clearing the first obstacle. This work passes the second hurdle.

We gave a full account on how to embed the KNP and HA suggestions in a string theory derived setting that takes into account moduli stabilization. We have used the $C_{2}$ axions as our would-be inflatons. The inflationary observables follow the pattern of the original natural inflation model. We have demonstrated that the hierarchy needed for moduli stabilization is the same hierarchy needed for the hierarchical axions scenario. Therefore, the KNP and HA scenarios are readily combined with moduli stabilization in a rather economical way.

Let us reiterate the main advantages of the models: First, inflation is generated only by nonperturbative effects, protecting it from perturbative corrections. Second, it requires the smallest number of axions. Third, there is no manifest tuning of the input parameters, since only simple hierarchies are necessary. Fourth, the inflationary trajectory is contained in a very small domain $\ll M_{p}$, again protecting it from dangerous corrections. This sheds light on the large field vs small field discussion, since it shows that the concern for large corrections due to large field excursions is important only if we consider the inflaton as a fundamental field, while for low energy effective fields, large field excursions are trivial and can be embedded in the constrained string theory setting. Last, it seems that the mathematical structure of the KNP/HA models has a broader context, and it will be interesting to investigate this structure thoroughly.

\section{ACKNOWLEDGMENTS}

This work is supported by the Impuls und Vernetzungsfond of the Helmholtz Association of German Research Centres under Grant No. HZ-NG-603, and the German Science Foundation (DFG) within the Collaborative Research Center 676 "Particles, Strings and the Early Universe." 
[1] G. Hinshaw et al., Nine-year Wilkinson Microwave Anisotropy Probe (WMAP) observations: Cosmological parameter results, Astrophys. J. Suppl. Ser. 208, 19 (2013).

[2] P. Ade et al. (Planck Collaboration), Planck 2013 results. XVI. Cosmological parameters, Astron. Astrophys. 571, A16 (2014).

[3] Z. Hou et al., Constraints on cosmology from the cosmic microwave background power spectrum of the 2500-square degree SPT-SZ survey, Astrophys. J. 782, 74 (2014).

[4] J. L. Sievers et al., The Atacama Cosmology Telescope: Cosmological parameters from three seasons of data, J. Cosmol. Astropart. Phys. 10 (2013) 060.

[5] P. Ade et al. (BICEP2 Collaboration), Detection of B-Mode Polarization at Degree Angular Scales by BICEP2, Phys. Rev. Lett. 112, 241101 (2014).

[6] P. Ade et al. (Planck Collaboration), Planck intermediate results. XIX. An overview of the polarized thermal emission from Galactic dust, Astron. Astrophys. 576, A104 (2015).

[7] R. Flauger, J. C. Hill, and D. N. Spergel, Toward an understanding of foreground emission in the BICEP2 region, J. Cosmol. Astropart. Phys. 08 (2014) 039.

[8] M. J. Mortonson and U. Seljak, A joint analysis of Planck and BICEP2 B modes including dust polarization uncertainty, J. Cosmol. Astropart. Phys. 10 (2014) 035.

[9] D. H. Lyth, What Would We Learn by Detecting a Gravitational Wave Signal in the Cosmic Microwave Background Anisotropy? Phys. Rev. Lett. 78, 1861 (1997).

[10] L. Boubekeur and D. Lyth, Hilltop inflation, J. Cosmol. Astropart. Phys. 07 (2005) 010.

[11] I. Ben-Dayan and R. Brustein, Cosmic microwave background observables of small field models of inflation, J. Cosmol. Astropart. Phys. 09 (2010) 007.

[12] S. Hotchkiss, A. Mazumdar, and S. Nadathur, Observable gravitational waves from inflation with small field excursions, J. Cosmol. Astropart. Phys. 02 (2012) 008.

[13] A. Hebecker, S. C. Kraus, and A. Westphal, Evading the Lyth bound in hybrid natural inflation, Phys. Rev. D 88, 123506 (2013).

[14] T. Banks, M. Dine, P. J. Fox, and E. Gorbatov, On the possibility of large axion decay constants, J. Cosmol. Astropart. Phys. 06 (2003) 001.

[15] E. Silverstein and A. Westphal, Monodromy in the CMB: Gravity waves and string inflation, Phys. Rev. D 78, 106003 (2008)

[16] L. McAllister, E. Silverstein, and A. Westphal, Gravity waves and linear inflation from axion monodromy, Phys. Rev. D 82, 046003 (2010).

[17] N. Kaloper and L. Sorbo, A Natural Framework for Chaotic Inflation, Phys. Rev. Lett. 102, 121301 (2009).

[18] N. Kaloper, A. Lawrence, and L. Sorbo, An ignoble approach to large field inflation, J. Cosmol. Astropart. Phys. 03 (2011) 023.

[19] E. Palti and T. Weigand, Towards large $r$ from $[p, q]-$ inflation, J. High Energy Phys. 04 (2014) 155.

[20] N. Kaloper and A. Lawrence, Natural chaotic inflation and UV sensitivity, Phys. Rev. D 90, 023506 (2014).

[21] F. Marchesano, G. Shiu, and A. M. Uranga, F-term axion monodromy inflation, J. High Energy Phys. 09 (2014) 184.
[22] R. Blumenhagen and E. Plauschinn, Towards universal axion inflation and reheating in string theory, Phys. Lett. B 736, 482 (2014).

[23] A. Hebecker, S. C. Kraus, and L. T. Witkowski, D7-brane chaotic inflation, Phys. Lett. B 737, 16 (2014).

[24] T. W. Grimm, Axion inflation in F-theory, Phys. Lett. B 739, 201 (2014).

[25] M. Dine, P. Draper, and A. Monteux, Monodromy inflation in SUSY QCD, J. High Energy Phys. 07 (2014) 146.

[26] L. McAllister, E. Silverstein, A. Westphal, and T. Wrase, The powers of monodromy, J. High Energy Phys. 09 (2014) 123.

[27] J. E. Kim, H. P. Nilles, and M. Peloso, Completing natural inflation, J. Cosmol. Astropart. Phys. 01 (2005) 005.

[28] M. Berg, E. Pajer, and S. Sjors, Dante's inferno, Phys. Rev. D 81, 103535 (2010).

[29] K. Choi, H. Kim, and S. Yun, Natural inflation with multiple sub-Planckian axions, Phys. Rev. D 90, 023545 (2014).

[30] T. Higaki and F. Takahashi, Natural and multi-natural inflation in axion landscape, J. High Energy Phys. 07 (2014) 074.

[31] R. Kappl, S. Krippendorf, and H. P. Nilles, Aligned natural inflation: Monodromies of two axions, Phys. Lett. B 737, 124 (2014).

[32] I. Ben-Dayan, F. G. Pedro, and A. Westphal, Hierarchical Axion Inflation, Phys. Rev. Lett. 113, 261301 (2014).

[33] S. H. H. Tye and S. S. C. Wong, Helical inflation and cosmic strings, arXiv:1404.6988.

[34] C. Long, L. McAllister, and P. McGuirk, Aligned natural inflation in string theory, Phys. Rev. D 90, 023501 (2014).

[35] X. Gao, T. Li, and P. Shukla, Combining universal and odd RR axions for aligned natural inflation, J. Cosmol. Astropart. Phys. 10 (2014) 048.

[36] T. Li, Z. Li, and D. V. Nanopoulos, Aligned natural inflation and moduli stabilization from anomalous $U(1)$ gauge symmetries, J. High Energy Phys. 11 (2014) 012.

[37] K. Freese, J. A. Frieman, and A. V. Olinto, Natural Inflation with Pseudo-Nambu-Goldstone Bosons, Phys. Rev. Lett. 65, 3233 (1990).

[38] X. Dong, B. Horn, E. Silverstein, and A. Westphal, Simple exercises to flatten your potential, Phys. Rev. D 84, 026011 (2011).

[39] S. B. Giddings, S. Kachru, and J. Polchinski, Hierarchies from fluxes in string compactifications, Phys. Rev. D 66, 106006 (2002).

[40] T. W. Grimm and J. Louis, The effective action of $N=1$ Calabi-Yau orientifolds, Nucl. Phys. B699, 387 (2004).

[41] T. W. Grimm, Non-perturbative corrections and modularity in $N=1$ type IIB compactifications, J. High Energy Phys. 10 (2007) 004.

[42] S. Kachru, R. Kallosh, A. D. Linde, and S. P. Trivedi, De Sitter vacua in string theory, Phys. Rev. D 68, 046005 (2003).

[43] V. Balasubramanian, P. Berglund, J. P. Conlon, and F. Quevedo, Systematics of moduli stabilisation in CalabiYau flux compactifications, J. High Energy Phys. 03 (2005) 007.

[44] C. Burgess, R. Kallosh, and F. Quevedo, De Sitter string vacua from supersymmetric D terms, J. High Energy Phys. 10 (2003) 056. 
[45] M. Cicoli, S. Krippendorf, C. Mayrhofer, F. Quevedo, and R. Valandro, D-branes at del Pezzo singularities: Global embedding and moduli stabilisation, J. High Energy Phys. 09 (2012) 019.

[46] M. Cicoli, A. Maharana, F. Quevedo, and C. Burgess, De Sitter string vacua from dilaton-dependent non-perturbative effects, J. High Energy Phys. 06 (2012) 011.

[47] D. Lust, S. Reffert, E. Scheidegger, W. Schulgin, and S. Stieberger, Moduli stabilization in type IIB orientifolds (II), Nucl. Phys. B766, 178 (2007).
[48] X. Gao and P. Shukla, F-term stabilization of odd axions in LARGE volume scenario, Nucl. Phys. B878, 269 (2014).

[49] M. Cicoli, K. Dutta, and A. Maharana, N-flation with hierarchically light axions in string compactifications, J. Cosmol. Astropart. Phys. 08 (2014) 012.

[50] T. W. Grimm, Axion inflation in type II string theory, Phys. Rev. D 77, 126007 (2008).

[51] J. P. Conlon, R. Kallosh, A. D. Linde, and F. Quevedo, Volume modulus inflation and the gravitino mass problem, J. Cosmol. Astropart. Phys. 09 (2008) 011. 